\title{
Numerical and Experimental Studies on Nonlinear Dynamics and Performance of a Bistable Piezoelectric Cantilever Generator
}

\author{
Kangkang Guo, ${ }^{1,2}$ Shuqian Cao, ${ }^{1,3}$ and Shiyu Wang ${ }^{1,3}$ \\ ${ }^{1}$ School of Mechanical Engineering, Tianjin University, Tianjin 300072, China \\ ${ }^{2}$ School of Measurement-Control Technology \& Communications Engineering, Harbin University of Science and Technology, \\ Harbin 150080, China \\ ${ }^{3}$ Tianjin Key Laboratory of Nonlinear Dynamics and Chaos Control, Tianjin 300072, China
}

Correspondence should be addressed to Shuqian Cao; sqcao@tju.edu.cn

Received 14 February 2015; Accepted 24 May 2015

Academic Editor: Roger Serra

Copyright (C) 2015 Kangkang Guo et al. This is an open access article distributed under the Creative Commons Attribution License, which permits unrestricted use, distribution, and reproduction in any medium, provided the original work is properly cited.

\begin{abstract}
A piezo-magneto-elastically coupled distributed-parameter model of a bistable piezoelectric cantilever generator is developed by using the generalized Hamilton principle. The influence of the spacing between two adjacent magnets on the static bifurcation characteristics of the system is studied and the range of magnet spacing corresponding to the bistable states is obtained. Numerical and experimental studies are carried out to analyze the bifurcation, response characteristics, and their impact on the electrical output performance under varying external excitations. Results indicate that interwell limit cycle motion of the beam around the two centers corresponds to optimum power output; interwell chaotic motion and multiperiodic motion including intrawell oscillations are less effective. At a given frequency, the phenomena of symmetric-breaking and amplitude-phase modulation are observed with increase of base excitation. Both period-doubling bifurcation and intermittency routes to chaotic motion in the bistable system are found. It can be observed that the power output is not proportional to the excitation level because of the bifurcation behaviours.
\end{abstract}

\section{Introduction}

Wireless sensor networks have been widely used in many fields, such as environmental monitoring, military defence, and medicine, in view of their convenience of signal transmission. As we know sensor nodes are in large quantities and located dispersedly. However, traditional power sources such as batteries are far from perfect for sensor nodes on account of the limited life and regular recharging. Besides, the power consumption of wireless sensor nodes is decreasing with the development of the MEMS technique, which makes the selfpowered nodes possible [1]. Vibration energy is ubiquitous in ambient environments, and, therefore, collecting vibration energy from the environment and converting it to usable electric energy has drawn a considerable amount of attention. The typical transduction from ambient vibration to electricity may be electrostatic, electromagnetic, or piezoelectric. Among them, piezoelectric transduction is regarded as the most hopeful way for MEMS devices due to the merits of the simple structure, high-energy density, and long lifetime, free from contamination and electromagnetic interference, as well as being easy to integrate [2].

Power generation of linear piezoelectric cantilever beams has been researched in many studies [3-6]. In order to enhance the power output, the linear cantilever system is required to work under resonance. As we know, it hardly matches the wide bandwidth and varying frequency characters of the ambient environment. To overcome this deficiency, some possible strategies have been proposed to widen the operational frequency range of the system and then improve the overall generating efficiency. For instance, piezoelectric generator arrays with a wider equivalent bandwidth $[7,8]$ and frequency tunable energy harvesters $[9,10]$ were presented. However, it is difficult to synchronize all oscillators in the array to reach maximum power output and integrate it into MEMS in view of its large volume. For the frequency tunable energy harvester, the automatic tuning is hard to realize in practice. 
In recent years, some researchers try to widen the operational frequency bandwidth using nonlinearities. Burrow et al. [11] indicated that cubic nonlinearity caused by the hardening stiffness effect in the vibration energy harvesters can be beneficial for the enhancement of operation bandwidth. Mann and Sims [12] designed a monostable nonlinear electromagnetic power generation device and observed the coexisting solutions in responses. By triggering the system jump to higher energy attractor, it is possible to improve its power generation capacity. Stanton et al. [13] proposed a monostable piezoelectric power generation beam which could exhibit both softening and hardening characteristics by tuning the interaction force between two magnets, and thus it can widen the bandwidth and maximize the power output. Nevertheless, in the multisolution region, it is only advantageous when the system is attracted to high-energy solutions, which is often difficult to realize.

In 2009, Gammaitoni et al. [14] introduced bistable configuration into vibration power generation and studied the impact of magnet spacing on the displacement and power output of the bistable structure. In the same year, Erturk et al. [15] implemented the bistable model proposed by Moon and Holmes [16] for piezoelectric power generation. Their researches show that the system can exhibit large amplitude periodic and chaotic oscillations excited by sinusoidal inputs. Compared with linear systems, the bistable system improves two times in voltage output and eight times in power output. Besides, Arrieta et al. [17] conducted experimental study on frequency response of a bistable piezoelectric plate, and various large amplitude oscillations, such as chaotic and large amplitude limit cycle motions which can obtain high voltages, were observed. Ferrari et al. [18] compared the displacement response of a bistable with a linear piezoelectric cantilever under band-limited white noise and verified the results by experiments. Ramlan et al. [19] investigated a snap-through mechanism and a hardening mechanism for energy harvesting devices and analyzed the advantage of each mechanism. Stanton et al. [20-22] investigated the performance of a bistable energy harvester by numerical and experimental methods. In their study, the phenomena of multiattractor coexistence and symmetry breaking of pitchfork bifurcation were observed. Since then, periodic and chaotic responses of the bistable power generation system were analyzed by different methods, such as harmonic balance method and Melnikov theory. Lin and Alphenaar [23] claimed that the power output of a piezoelectric cantilever driven by random noise source can be enhanced by introducing a repulsive magnetic force. Sun and Cao [24] presented the modeling of a bistable piezoelectric generator and studied the response characteristics of the system. Tang et al. [25] investigated both monostable and bistable configurations of a nonlinear energy harvesting device subjected to harmonic and random excitations and pointed out that the optimal performance exists near the transition region. McInnes et al. [26] proposed a new approach to improve the performance of nonlinear energy harvester using stochastic resonance. Two reviews on the researches of bistable vibration energy harvesters have been published in recent two years [27, 28].
The present studies indicate the potential of bistable power generators is having interwell oscillations in a wider low-frequency region. However, complex nonlinear dynamic behavior provides bigger challenges for the improving of the power generation performance and the designing of the conditioning circuit. Therefore, it is necessary to ascertain the system's dynamic bifurcation characteristic and its impact on the power generation and obtain the occurrence condition of high-energy orbits. In the present paper, the piezo-magnetoelastically coupled distributed-parameter model for a bistable piezoelectric cantilever generator (BPCG) is established by using the generalized Hamilton principle firstly. Based on the model, static bifurcation analysis is carried out to detect the magnet spacing range corresponding to the bistable state. Subsequently, numerical simulations and experimental validations are performed to illustrate the influence of some crucial parameters of the system on the nonlinear dynamic responses, the bifurcation characteristics, and the output performance.

\section{Description and Modelling of the BPCG}

In this section, piezo-magneto-elastically coupled model is derived for describing the behaviours of the BPCG. A simplified physical model is shown in Figure 1. It is a nonlinear oscillator comprised of a base-excited piezoelectric cantilever with an adjustable magnetic force at the free end. The piezoelectric cantilever is an elastic beam with a piezoelectric patch bonded on the upper surface near the fixed-end of the cantilever. One permanent magnet $A$ is mounted at the tip of the beam, and the other magnet $B$ is mounted on the base at a distance of $d$ from $A$. The two magnets are placed with opposite magnetization to provide repulsive force. The polarization direction of the piezoelectric ceramic is along direction 3. Physical coordinates are denoted by $X$ and $Y$.

The constitutive relationship of the elastic beam is

$$
T_{1}=c_{11}^{s} S_{1}
$$

where $T_{1}$ and $S_{1}$ denote the mechanical stress and strain along direction 1 , respectively, and $c_{11}^{s}$ is the substrate stiffness coefficient. For the piezoelectric patch, the constitutive relationship with piezoelectric effect can be expressed as

$$
\begin{aligned}
& T_{1}=c_{11}^{E} S_{1}-e_{31} E_{3}, \\
& D_{3}=\varepsilon_{33}^{S} E_{3}+e_{31} S_{1},
\end{aligned}
$$

where $E_{3}$ and $D_{3}$ are the electric field and displacement along direction 3 , respectively, $c_{11}^{E}$ is the elastic stiffness at zero electric field, $e_{31}$ is the piezoelectric coefficient, and $\varepsilon_{33}^{S}$ represents the permittivity constant at zero strain.

2.1. Piezomagnetoelastic Model. The cantilever oscillates transversely under base excitation, while the two magnets produce nonlinear repelling force. According to the 


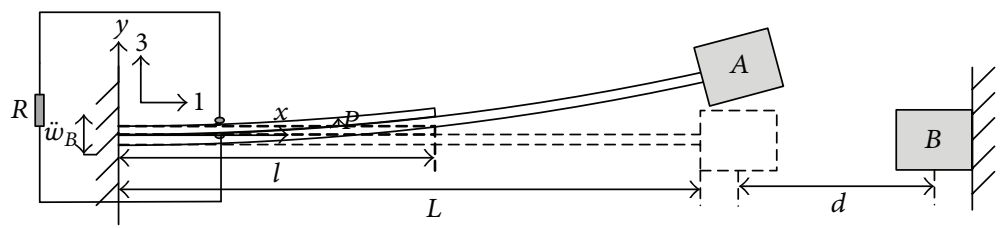

FIGURE 1: Schematic of the bistable piezoelectric cantilever generator.

generalized Hamilton variation principle, a piezomagnetoelastic system can be modelled as follows:

$$
\int_{t_{1}}^{t_{2}}\left[\delta\left(T_{k}-U+W_{e}\right)+\delta W-\delta U_{m}\right] \mathrm{d} t=0,
$$

where $T_{k}$ is the system kinematic energy, $U$ is the potential energy, $W_{e}$ is the piezoelectric ceramic electrical energy, $\delta W$ is the variation of external work, and $\delta U_{m}$ is the variation of the repulsion energy, for which detailed expression is given in next section. Their expressions are given as follows:

$$
\begin{aligned}
T_{k}= & \frac{1}{2} \int_{V_{s}} \rho_{s} \dot{u}^{2}(X, t) \mathrm{d} V_{s}+\frac{1}{2} \int_{V_{p}} \rho_{p} \dot{u}^{2}(X, t) \mathrm{d} V_{p} \\
& +\frac{1}{2} m_{0} \dot{u}^{2}(L, t), \\
U= & \frac{1}{2} \int_{V_{s}} S_{1} T_{1} \mathrm{~d} V_{s}+\frac{1}{2} \int_{V_{p}} S_{1} T_{1} \mathrm{~d} V_{p}, \\
W_{e}= & \frac{1}{2} \int_{V_{p}} E_{3} D_{3} \mathrm{~d} V_{p},
\end{aligned}
$$

where $\rho$ is the material density, $V$ is the volume, and subscripts $s$ and $p$ indicate the elastic beam (substrate) and the piezoelectric layer, respectively. $u(X, t)$ denotes the transverse displacement of the beam; $m_{0}$ is the mass of the magnet, and the notation "." indicates a time derivative.

Assuming the beam's mass per unit length is $m(X)$, and then the variation of external work can be written as

$$
\delta W=-\int_{0}^{L} m(X) \ddot{w}_{B} \delta u d X-m_{0} \ddot{w}_{B} \delta u(L, t)+\delta \varphi q,
$$

where $\varphi$ is the scalar electrical potential and $q$ the quantity of electrical charge.

Considering one mode of the beam and regarding the electrodes on the lower and upper surface of the piezoelectric layer as a single electrode pair, then we have the following expressions based upon Rayleigh-Ritz method, Euler-Bernoulli beam theory, and constant electrical field assumption of piezoelectric element:

$$
\begin{aligned}
u(x, t) & =\psi_{r}(x) r(t), \\
S_{1} & =-y \frac{\partial^{2} u(x, t)}{\partial x^{2}}=-y \psi_{r}^{\prime \prime} r(t), \\
E_{3} & =-\frac{\partial \psi_{v}(y)}{\partial y} v(t)=-\psi_{v}^{\prime} v(t)=\frac{1}{t_{p}} v(t),
\end{aligned}
$$

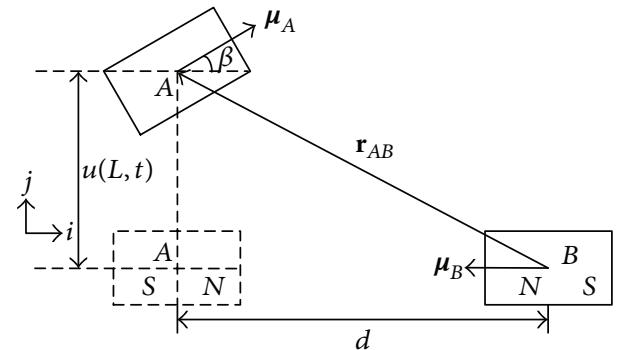

FIGURE 2: Schematic of the two permanent magnets in repelling mode.

where $\psi_{r}(X)$ represents the first modal of bending; $r(t)$ is the modal coordinate of transverse vibration; $\psi_{v}(X)$ is the electrical potential distribution; $v(t)$ is the generalized voltage modal coordinate; $t_{p}$ is the piezoelectric layer thickness, and the notation " " " indicates the derivative with respect to $X$.

2.2. Magnetic Repulsion Energy Model. Figure 2 shows the interaction force of the two rectangular permanent magnets. Under the repelling force, permanent magnet $A$, which is mounted at the free end of the beam, leaves its horizontal position with a displacement $u(L, t)$ and deflection angle $\beta=$ $u^{\prime}(L, t)$. The instantaneous distance vector between the mass centres of the two magnets can be expressed as

$$
\mathbf{r}_{A B}=-d \cdot \mathbf{i}+u(L, t) \cdot \mathbf{j} .
$$

Regarding the two magnets as point dipoles, both of the magnetic dipole moments are

$$
\begin{aligned}
& \boldsymbol{\mu}_{A}=M_{A} V_{A} \cos \beta \mathbf{i}+M_{A} V_{A} \sin \beta \mathbf{j}, \\
& \boldsymbol{\mu}_{B}=-M_{B} V_{B} \mathbf{i},
\end{aligned}
$$

where $M_{A}$ and $M_{B}$ are the magnified strength of permanent magnets $A$ and $B$, and $V_{A}$ and $V_{B}$ are the volumes of two magnets, respectively. Magnetic field at $B$ generated by $A$ can be calculated by vector differentiation approach $[20,29]$ :

$$
\mathbf{B}_{A B}=-\frac{\mu_{0}}{4 \pi} \nabla \frac{\boldsymbol{\mu}_{A} \mathbf{r}_{A B}}{\left\|\mathbf{r}_{A B}\right\|_{2}^{3}}
$$


where $\mu_{0}=4 \pi \times 10^{-7}$ is the permeability in vacuum and $\nabla$ and $\|\cdot\|_{2}$ are the vector gradient operator and Euclid norm, respectively. With these expressions, one can have the potential of the two magnets as follows:

$$
\begin{aligned}
U_{m} & =-\mathbf{B}_{A B} \cdot \boldsymbol{\mu}_{B} \\
& =\frac{-\mu_{0} M_{A} V_{A} M_{B} V_{B}}{4 \pi}\left[\frac{1-(1 / 2) u^{\prime 2}(L, t)}{\left(d^{2}+u^{2}(L, t)\right)^{3 / 2}}\right.
\end{aligned}
$$

$$
\left.+\frac{3 d^{2}\left((1 / 2) u^{\prime 2}(L, t)-1\right)+3 d u(L, t) u^{\prime}(L, t)}{\left(d^{2}+u^{2}(L, t)\right)^{5 / 2}}\right] .
$$

Considering (6) and expanding (12) at $r=0$ using Taylor expansion, then one can have

$$
\delta U_{m}=\left(-K_{1} r+K_{2} r^{3}+O\left(r^{5}\right)\right) \delta r,
$$

where

$$
\begin{aligned}
& K_{1}=\frac{\mu_{0} M_{A} V_{A} M_{B} V_{B}}{2 \pi}\left[\frac{d^{2} \psi_{r}^{\prime 2}(L)+6 \psi_{r}^{2}(L)+3 d \psi_{r}(L) \psi_{r}^{\prime}(L)}{d^{5}}\right], \\
& K_{2}=\frac{3 \mu_{0} M_{A} V_{A} M_{B} V_{B}}{8 \pi}\left[\frac{30 \psi_{r}^{4}(L)+13 d^{2} \psi_{r}^{2}(L) \psi_{r}^{\prime 2}(L)+20 d \psi_{r}^{3}(L) \psi_{r}^{\prime}(L)}{d^{7}}\right] .
\end{aligned}
$$

2.3. Simplification and Nondimensionalization of Equations. Substituting (4), (5), and (13) into (3), and considering (6)(8), one can have

$$
\begin{aligned}
M \ddot{r}+C \dot{r}+K r-K_{1} r-\theta v+K_{2} r^{3} & =-\Gamma_{B} \ddot{w}_{B}, \\
\theta r+C_{p} v+q & =0,
\end{aligned}
$$

where $M$ and $K$ are the modal mass and modal stiffness of the beam oscillator; $\theta$ and $C_{p}$ are the electromechanical coupling coefficient and capacitance of the piezoelectric element, and $\Gamma_{B}$ is the external excitation coefficient. Moreover, it should be noted that mechanical damping is included by adding a viscous damping term:

$$
\begin{aligned}
M & =\int_{V_{s}} \rho_{s} \psi_{r}^{2} \mathrm{~d} V_{s}+\int_{V_{p}} \rho_{p} \psi_{r}^{2} \mathrm{~d} V_{p}+m_{0} \psi_{r}^{2}(L), \\
K & =\int_{V_{s}} c_{11}^{s} y^{2} \psi_{r}^{\prime \prime 2} \mathrm{~d} V_{s}+\int_{V_{p}} c_{11}^{E} y^{2} \psi_{r}^{\prime \prime 2} \mathrm{~d} V_{p}, \\
\theta & =\int_{V_{p}} e_{31} \psi_{r}^{\prime \prime} y \psi_{v}^{\prime} \mathrm{d} V_{p}, \\
C_{p} & =\int_{V_{p}} \varepsilon_{33}^{S} \psi_{v}^{2} \mathrm{~d} V_{p}, \\
\Gamma_{B} & =m \int_{0}^{L} \psi_{r} \mathrm{~d} x+m_{0} \psi_{r}(L) .
\end{aligned}
$$

Assume the base excitation as

$$
\ddot{w}_{B}=Z_{e} \cos \left(\Omega_{e} t\right),
$$

where $Z_{e}$ is the acceleration amplitude of the base and $\Omega_{e}$ is the excitation (angular) frequency in $\mathrm{rad} / \mathrm{s}$. Assume the electrical load as a pure resistance $R_{L}$, and thus the voltage is $v=(\mathrm{d} q / \mathrm{d} t) \cdot R_{L}$. Hence, (15) can be rewritten as

$$
\begin{aligned}
\ddot{r}+ & 2 \zeta_{m} \omega_{1} \dot{r}+\omega_{1}^{2} r-\frac{K_{1}}{M} r-\frac{\theta}{M} v+\frac{K_{2}}{M} r^{3} \\
& =-\frac{\Gamma_{B} Z_{e}}{M} \cos \left(\Omega_{e} t\right), \\
\theta \dot{r} & +C_{p} \dot{v}+\frac{1}{R_{L}} v=0,
\end{aligned}
$$

where $\omega_{1}=\sqrt{K / M}$ and $\zeta_{m}=C /\left(2 M \omega_{1}\right)$ is the damping ratio which is estimated according to [4].

By introducing the dimensionless transformations: $x=$ $r / L, u=v / v c, \tau=\omega_{1} t$, and $v_{c}=L \theta / C_{p}$, then (18) are expressed in a dimensionless and simplified form as

$$
\begin{aligned}
\ddot{x}+2 \zeta_{m} \dot{x}+x-\kappa_{1} x-\vartheta^{2} u+\kappa_{2} x^{3} & =f \cos (\omega \tau), \\
\dot{u}+\dot{x}+\alpha u & =0,
\end{aligned}
$$

where

$$
\begin{aligned}
\vartheta^{2} & =\frac{\theta^{2}}{K C_{p}}, \\
\kappa_{1} & =\frac{K_{1}}{K}, \\
\kappa_{2} & =\frac{K_{2} L^{2}}{K}, \\
\omega & =\frac{\Omega_{e}}{\omega_{1}}, \\
\alpha & =\frac{1}{R_{L} C_{p} \omega_{1}}, \\
f & =-\frac{\Gamma_{B} Z_{e}}{K L} .
\end{aligned}
$$


TABLE 1: Geometric and material properties of the bistable piezoelectric cantilever generator.

\begin{tabular}{lc}
\hline Parameter & Value \\
\hline Elastic beam length $L(\mathrm{~mm})$ & 75 \\
Elastic beam width $b(\mathrm{~mm})$ & 22 \\
Elastic beam density $\rho_{s}\left(\mathrm{~kg} / \mathrm{m}^{3}\right)$ & 8500 \\
Elastic beam thickness $t_{s}(\mathrm{~mm})$ & 0.38 \\
Substrate stiffness $c_{11}^{S}(\mathrm{GPa})$ & 47 \\
Cantilever beam mass $M_{c}(\mathrm{~g})$ & 14.57 \\
Magnet mass $m_{0}(\mathrm{~g})$ & 14.9 \\
Magnet volume $V_{A}, V_{B}\left(\mathrm{~mm}^{3}\right)$ & $2 \times 10^{3}$ \\
Residual flux density $(\mathrm{T})$ & 1.2 \\
Piezoelectric patch length $l(\mathrm{~mm})$ & 40 \\
Piezoelectric patch width $b_{0}(\mathrm{~mm})$ & 20 \\
Piezoelectric patch density $\rho_{p}\left(\mathrm{~kg} / \mathrm{m}^{3}\right)$ & 7100 \\
Piezoelectric patch thickness $t_{p}(\mathrm{~mm})$ & 0.12 \\
Piezoelectric patch stiffness $c_{11}^{E}(\mathrm{GPa})$ & 60 \\
Absolute permittivity $\varepsilon_{33}^{S}(\mathrm{~F} / \mathrm{m})$ & $3500 \varepsilon_{0}$ \\
Vacuum permittivity $\varepsilon_{0}\left(10^{-12} \mathrm{~F} / \mathrm{m}\right)$ & 8.854 \\
Piezoelectric coefficient $e_{31}\left(\mathrm{C} / \mathrm{m}^{2}\right)$ & -16.5 \\
Loading resistance $R_{L}(\mathrm{k} \Omega)$ & 91 \\
\hline
\end{tabular}

TABLE 2: Key parameters in the mathematical model.

\begin{tabular}{lc}
\hline Model parameter & Value \\
\hline$M(\mathrm{~kg})$ & 0.12 \\
$K(\mathrm{~N} / \mathrm{m})$ & 7150 \\
$\theta(\mathrm{N} / \mathrm{V})$ & -0.0066 \\
$C_{p}(\mathrm{~F})$ & $4.5 \times 10^{-8}$ \\
$\Gamma_{B}(\mathrm{~kg})$ & 0.061 \\
$\zeta_{m}$ & 0.015 \\
$\vartheta^{2}$ & 0.1613 \\
$\Psi_{r}(L)$ & 1.997 \\
\hline
\end{tabular}

2.4. Static Bifurcation Analysis. It can be seen from (14a), (14b), and (21) that when the structural and magnetic parameters are fixed, variation of the magnet spacing $d$ can lead to the change of stiffness $\kappa_{1}$ and stiffness $\kappa_{2}$. The influence of the magnet spacing on the static bifurcation characteristics of the system can be investigated from energy perspective; the potential energy of the system under the short-circuit condition can be written as

$$
\bar{V}=-\left(\kappa_{1}-1\right) \frac{x^{2}}{2}+\kappa_{2} \frac{x^{4}}{4} .
$$

In order to study the influence of $d$, geometric parameters and material properties of the system are given in Table 1 and the key effective parameters of the mathematical model are listed in Table 2. Figure 3 shows the relationship of $\kappa_{1}$ and $\kappa_{2}$ with respect to $d$. It can be observed that both $\kappa_{1}$ and $\kappa_{2}$ decrease but at different rate with an increase of $d$. Note that when $d=14.22 \mathrm{~mm}$ and $\kappa_{1}=1$, the coefficient of linear stiffness in (19) is zero. The topological properties in phase space and the shape of the potential energy curve are all changed when crossing the critical value

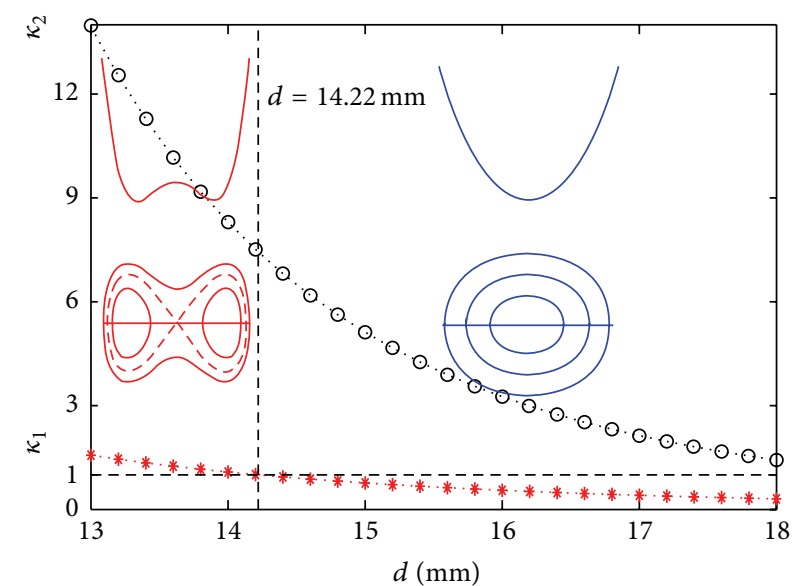

FIgURE 3: Change of $\kappa_{1}$ and $\kappa_{2}$ with respect to $d$, and the topological shape of the potential energy curves and phase portraits corresponding to both sides of the bifurcation point.

$(d=14.22 \mathrm{~mm})$ (Figure 3$)$. In the case of $d>14.22 \mathrm{~mm}$ and $\kappa_{1}<1$, the system has positive linear stiffness, and there is one well on the potential energy curve corresponding to the equilibrium at $(0,0,0)$, the system is monostable; when $d<14.22 \mathrm{~mm}$ and $\kappa_{1}>1$, the system has two potential wells and one potential barrier, which correspond to the two stable equilibriums (centres) at $\left( \pm \sqrt{\left(\kappa_{1}-1\right) / \kappa_{2}}, 0,0\right)$ and one unstable equilibrium (saddle point) at $(0,0,0)$. The potential well depth is $\left(\kappa_{1}-1\right)^{2} / 4 \kappa_{2}$. The above analysis shows that the supercritical pitchfork bifurcation occurs with the change of $d$. Proper magnet spacing is the key factor for the bistability of the system. In the following sections, the dynamic response and power generation performance of the BPCG are investigated by numerical and experimental methods.

\section{Numerical Simulations}

The major objective of this section is to investigate the features of the dynamic response and voltage output of the BPCG by numerical simulations. Set $d=13.5 \mathrm{~mm}$, the system exhibits the bistable case, and the initial conditions are assumed to be $(x(0), \dot{x}(0), u(0)=(0.15,0,0))$ (without specific notification, hereinafter the same initial values). Equations (19) and (20) are solved as the excitation frequency or amplitude is varied.

3.1. Effect of Excitation Frequency. A bifurcation diagram of the BPCG, while fixing the excitation amplitude at $Z_{e}=2.5 \mathrm{~g}$ ( $g=9.8 \mathrm{~m} / \mathrm{s}^{2}$, the gravitational acceleration) and increasing frequency $\omega_{e}\left(\omega_{e}=\Omega_{e} / 2 \pi\right)$, is showed in Figure 4. It can be seen that the bistable system exhibits rich dynamics under base excitations. As the excitation frequency increases, chaotic and periodic motions occur interactively. The system responses can be categorized into intrawell and interwell motions, where interwell motion can be further classified as large amplitude limit cycle oscillations, chaotic motions, 


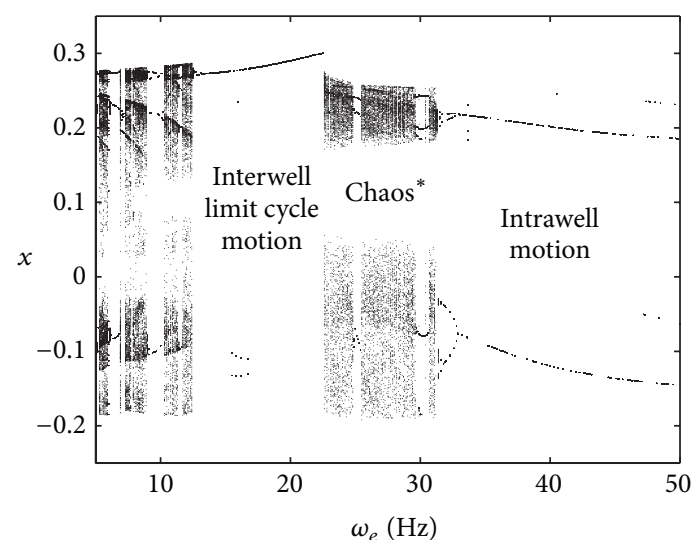

FIgURE 4: Bifurcation diagram as the excitation frequency $\omega_{e}$ is increased for $Z_{e}=2.5 \mathrm{~g}$, where “*” indicates chaotic region, includes several periodic windows.

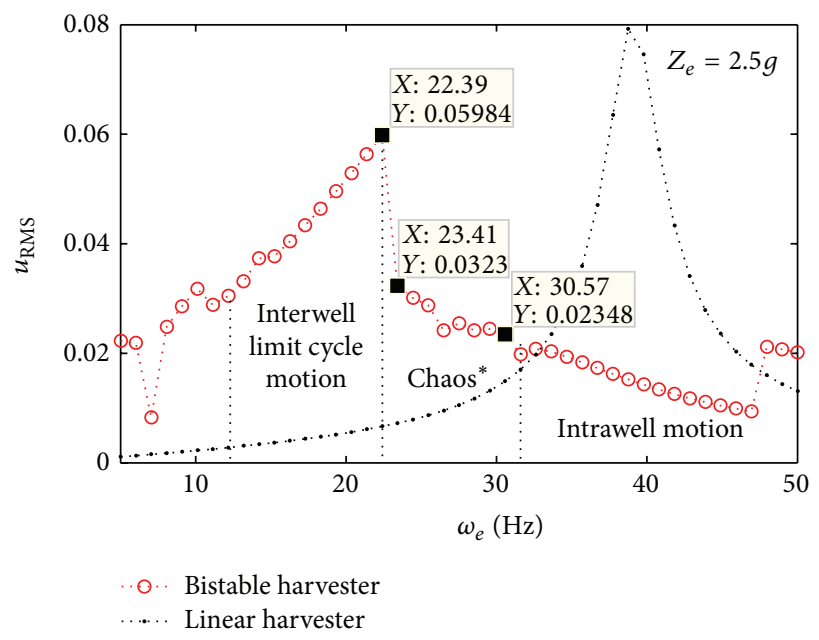

FIGURE 5: Numerically obtained effective voltage outputs during frequency sweep of the bistable harvester versus the linear harvester for $Z_{e}=2.5 \mathrm{~g}$.

and large amplitude multiple periodic motions containing intrawell oscillation.

In order to find out which form of the above-mentioned interwell motions can bring the optimal voltage output, we calculate the steady state electrical response by MATLAB simulation. With fixed excitation amplitude of $2.5 \mathrm{~g}$, Figure 5 gives the effective value of voltage output while increasing frequency from $5 \mathrm{~Hz}$ to $50 \mathrm{~Hz}$ at the interval of $1 \mathrm{~Hz}$. Within the low frequency range, interwell motion dominates the response of the bistable system, which shows obvious larger power generated in the nonlinear system than in the linear system, and the large amplitude limit cycle motion corresponds to the optimal power output. In addition, effective values of output voltage increase with increasing excitation frequency. Compared with the large amplitude limit cycle motion, the voltage output is low when the system is chaotic or large amplitude multiple periodic $(23.41 \mathrm{~Hz} \sim 31.59 \mathrm{~Hz})$ (Figure 5). Moreover, the voltage output is independent of the excitation frequency. Taking the three typical interwell motions (large amplitude limit cycle oscillation and chaotic and period-5 motion) under $Z_{e}=2.5 \mathrm{~g}$ as examples, Figure 6 gives the time and frequency domain responses of the displacement and voltage. It can be found that the system has optimal power output under interwell, large orbit oscillations around the three equilibrium points; period-5 motion contains intrawell and interwell motions, where the power output is obviously reduced; when the system response behaves chaotically, due to the intrinsic randomness of chaos, intrawell and interwell motions appear interactively, and thus the power output is also chaotic but the effective value is low. In short, with the same excitation amplitude, there exists a frequency range of the excitation, which is identified to be the optimal region of power output, corresponding to large amplitude limit cycle oscillations.

3.2. Effect of Excitation Amplitude. From Figures 6(a) and $6(\mathrm{c})$, it is observed that the phase trajectories of the periodic responses are symmetric. This can also be verified by (19) and (20), where the form of equations remains invariant under the transformation of $x \rightarrow-x, u \rightarrow-u$, and $\tau \rightarrow \tau+(\pi / \omega)$. However, the symmetric property will disappear under certain conditions, as shown in Figure 7, for $\omega_{e}=12 \mathrm{~Hz}$ and $Z_{e}=2 \mathrm{~g}$. This is because under a fixed excitation frequency, the nonlinear frequency increases fast with increasing excitation amplitude. When the nonlinear frequency is close to $2 n \omega$, the periodic solution includes both odd and even superharmonics, which results in the loss of symmetry, namely, symmetry-breaking bifurcation. If the excitation keeps unchanged, dual solutions appear for different initial conditions (Figures 7(a) and 7(b)). The appearance of dual solutions is derivative from symmetrybreaking bifurcation [30]. The above analysis indicates that the asymmetry of periodic orbit discovered in this work is caused by the system nonlinearity, whereas the symmetrybreaking behaviour causing an imperfect pitchfork bifurcation described in [20] is attributed to the positional and angular offsets of the right-side magnet, which is quite different from the symmetry-breaking bifurcation mentioned here. The symmetry-breaking bifurcation usually precedes the period-doubling bifurcation. With a gradual increase of $Z_{e}$, a series of period-doubling bifurcations occur. Figures 7 (c) and 7(d) show period-2 and period-4 motions, respectively. As $Z_{e}$ reaches $2.1 \mathrm{~g}$, chaotic motion happens; Figure 8(a) shows the phase portrait and Poincaré section corresponding to the chaotic motion, which has the maximum Lyapunov exponent of 0.0287 when the system is at steady state (Figure $8(\mathrm{~b})$ ).

When $Z_{e}$ is increased to $2.12 \mathrm{~g}$, the system response appears as irregular chaotic bursts in addition to the periodic behaviour, as shown in Figure 9(a). As $Z_{e}$ is further increased, chaotic burst becomes more frequent, as shown in Figure 9(b), and finally the regular motion almost disappears and the system is fully chaotic (Figure 10). The results suggest that the system has another typical route to chaos, namely, intermittency.

The analysis indicates that there are two typical routes to chaos (periodic-doubling and intermittency) which occurred in the BPCG. Figure 11 depicts the bifurcation diagram with 

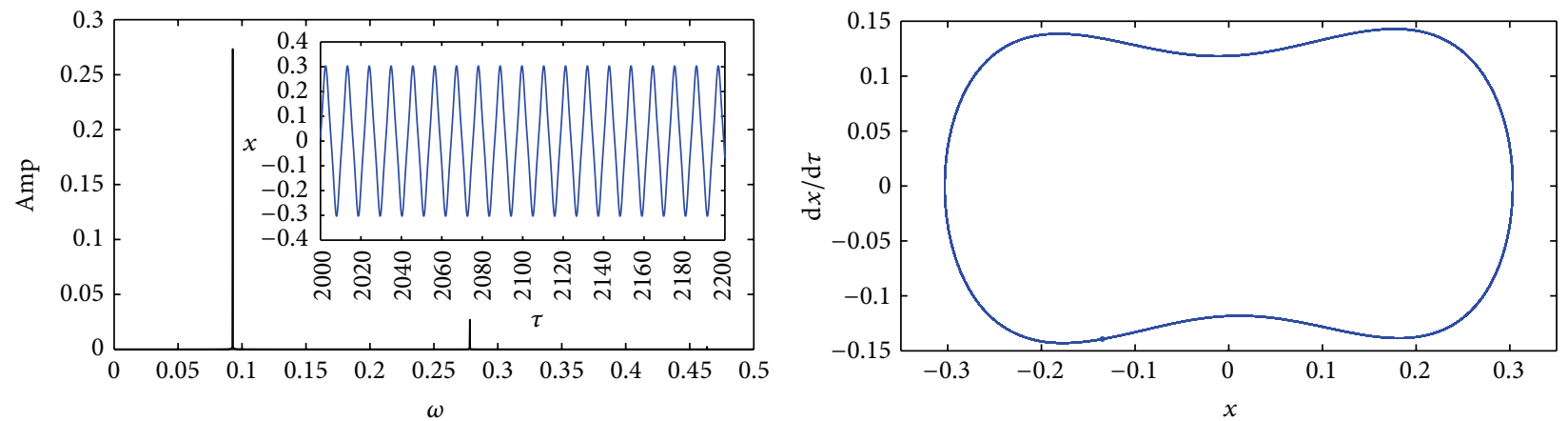

(a) Period-one motion at $\omega_{e}=22.39 \mathrm{~Hz}$
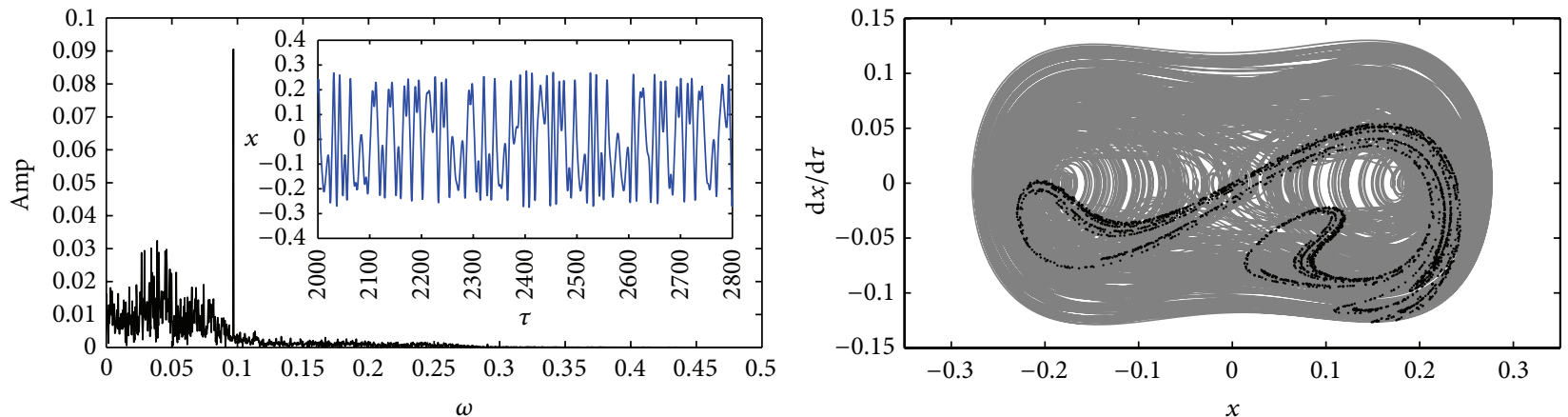

(b) Chaotic motion at $\omega_{e}=23.41 \mathrm{~Hz}$
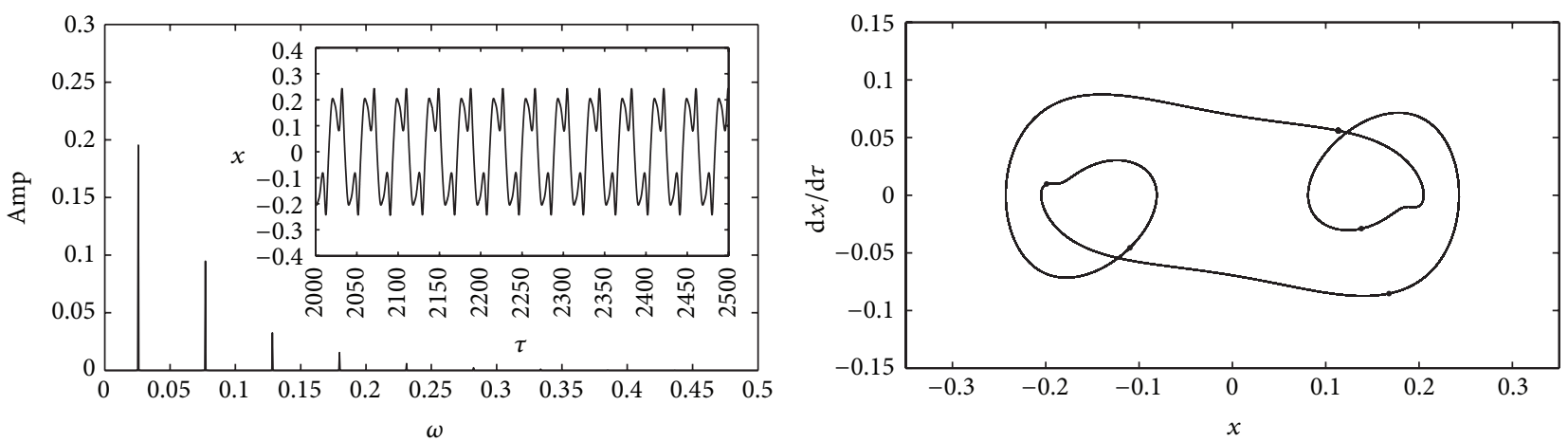

(c) Period-five motion at $\omega_{e}=30.57 \mathrm{~Hz}$

FIGURE 6: System response obtained numerically with different excitation frequency. Left: time series and frequency spectrum; right: phase trajectory and Poincaré maps.

increasing $Z_{e}$ for $\omega_{e}=12 \mathrm{~Hz}$, as an evidence of the coexistence of two routes to chaos. From the locally enlarged figure, one can find the discontinuity within the bifurcation region. This is because the symmetrical attractor will be replaced by two coexisting asymmetric attractors when symmetrybreaking bifurcation occurs [30]. In different initial conditions, the phase portraits of the dual solutions mirrored each other (see Figures 7(a) and 7(b)). Similar phenomenon will happen when the excitation amplitude changes. Comparing Figures 12 with 7 (a), we can see that the amplitude-phasemodulation can be discovered with $Z_{e}=2 g$ and $2.02 g$ under the same initial condition. This can be further observed by the discontinuous jumping phenomenon in the bifurcation diagram (see Figure 11(b) for an enlarged view). Accordingly, Figure 13 gives the changes of effective voltage output with respect to increase of the excitation amplitude. Power output is not always increasing with the increase of the input energy due to the bifurcation. It should be noted that, in the state of the period-doubling bifurcation and the beginning of intermittency, power output keeps higher due to the fact that interwell motion is dominated and does not change significantly. However, when the response becomes fully chaotic, the power output reduces remarkably.

Based upon the above analysis, when designing a BPCG, the optimal system output can be acquired by postponing the bifurcation occurrence, suppressing the chaotic motion and extending the frequency bandwidth of large amplitude periodic oscillations.

\section{Experimental Investigations}

4.1. Experimental Setup and Procedures. The experimental layout is showed in Figure 14(a) and a picture of the piezomagnetoelastic vibration power generator device 


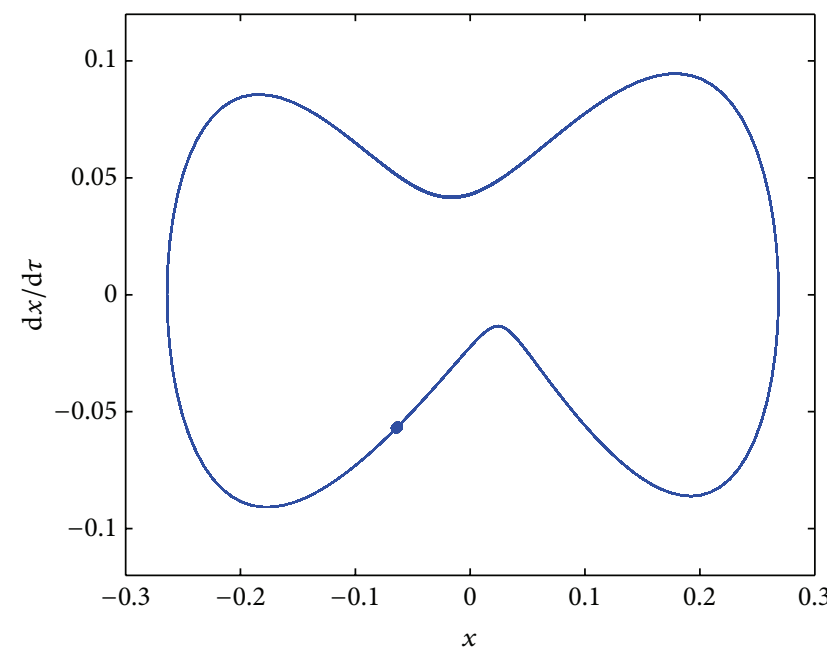

(a)

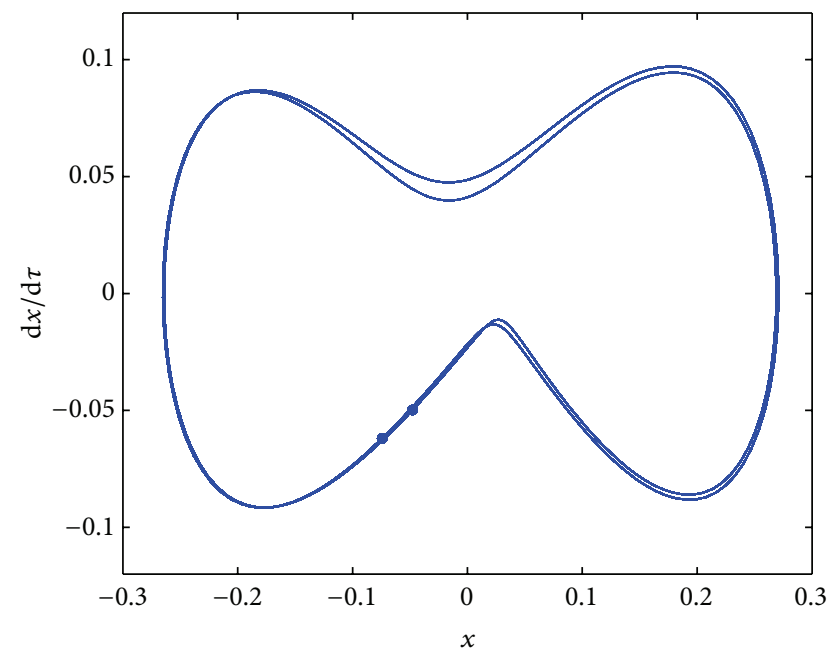

(c)

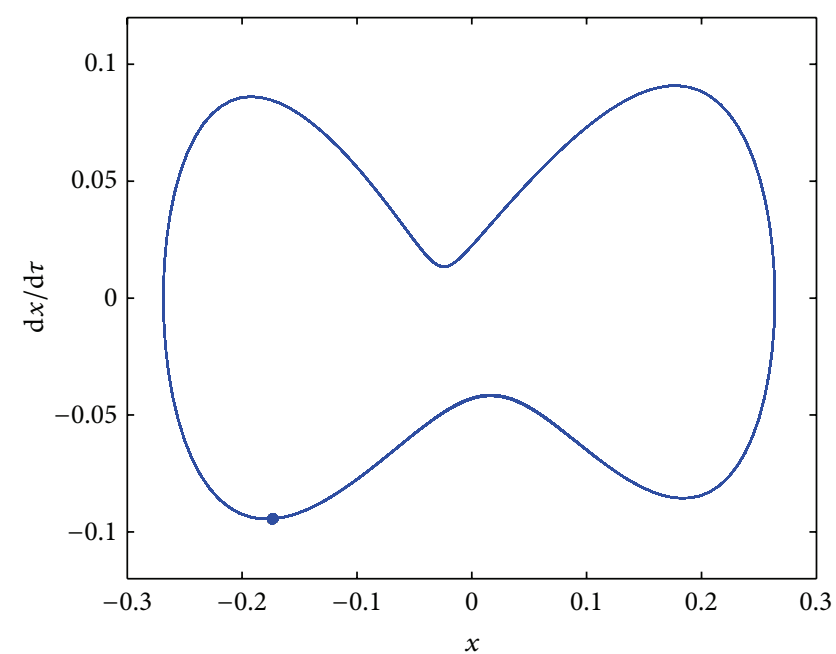

(b)

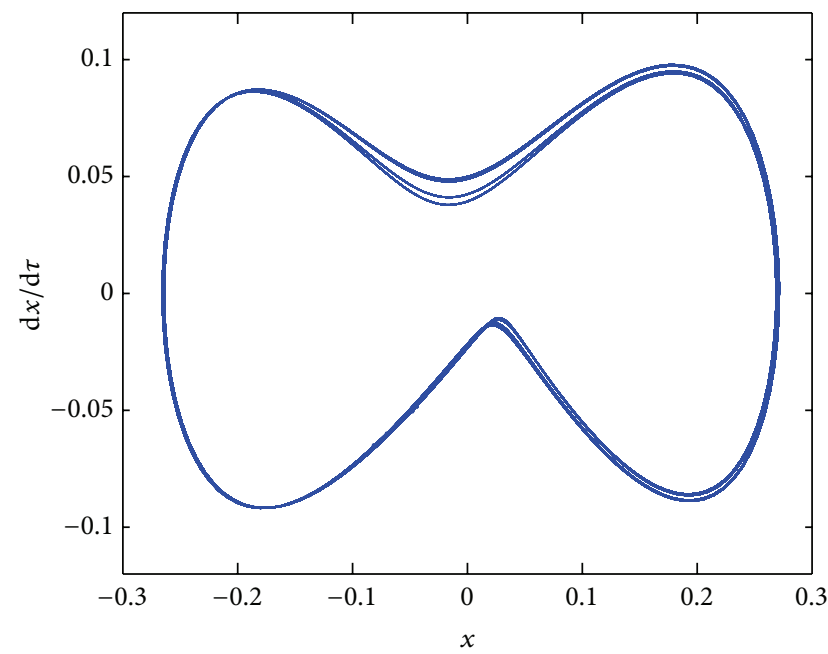

(d)

Figure 7: Phase trajectory and Poincaré maps for $\omega_{e}=12 \mathrm{~Hz}$ with increasing $Z_{e}$, where (a) asymmetric period-1 solution, $Z_{e}=2 g$; (b) dual solution, $Z_{e}=2 \mathrm{~g}$, with different initial conditions $(-0.5,0,0)$; (c) period-2 solution, $Z_{e}=2.06 \mathrm{~g}$; and (d) period- 4 solution, $Z_{e}=2.07 \mathrm{~g}$.

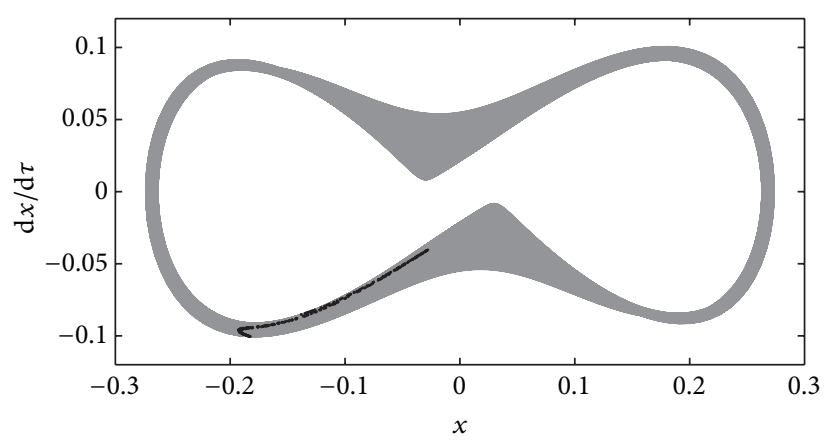

(a)

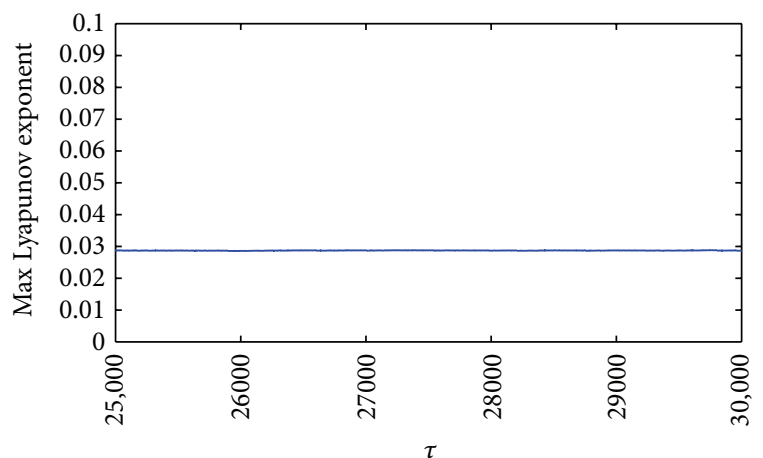

(b)

FIGURE 8: Response of the system with $\omega_{e}=12 \mathrm{~Hz}$ and $Z_{e}=2.1 \mathrm{~g}$ : (a) phase plot and Poincaré section and (b) the max Lyapunov exponents. 


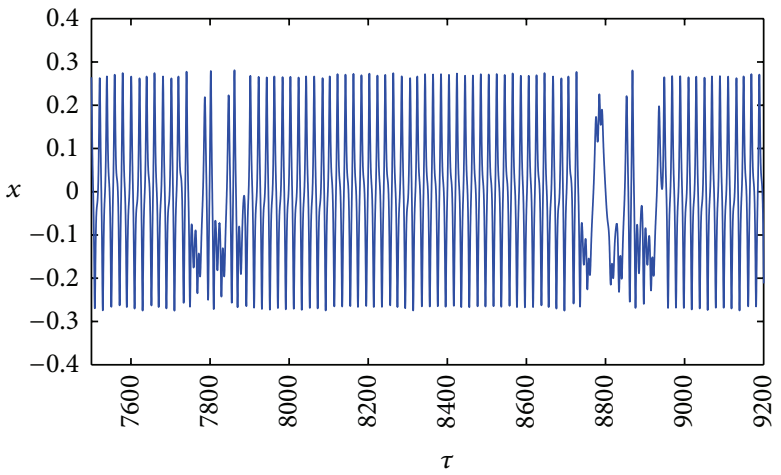

(a)

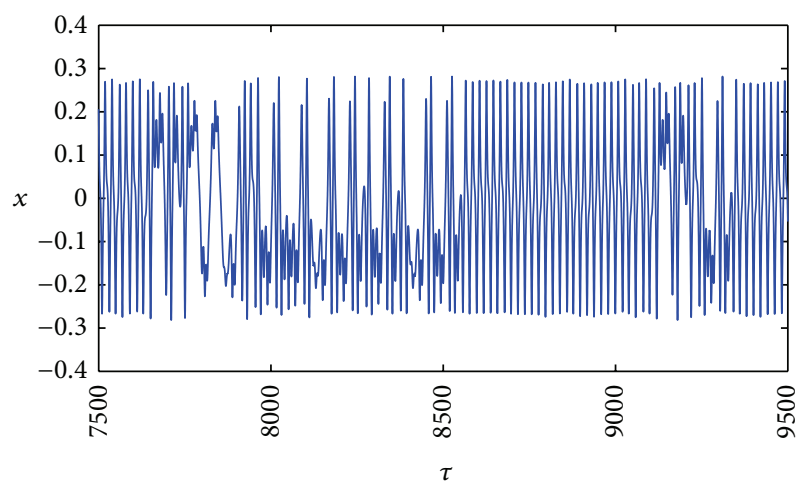

(b)

FIGURE 9: Numerically obtained time series of the vibration response for $\omega_{e}=12 \mathrm{~Hz}$ and (a) $Z_{e}=2.12 \mathrm{~g}$ and (b) $Z_{e}=2.13 \mathrm{~g}$.

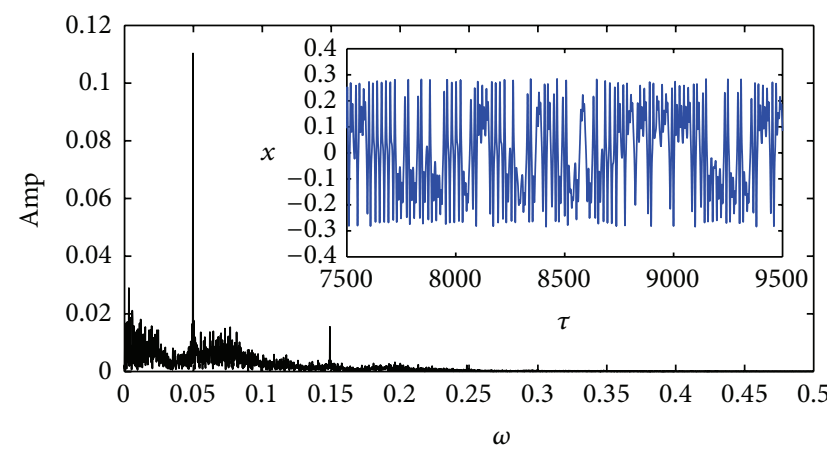

(a)

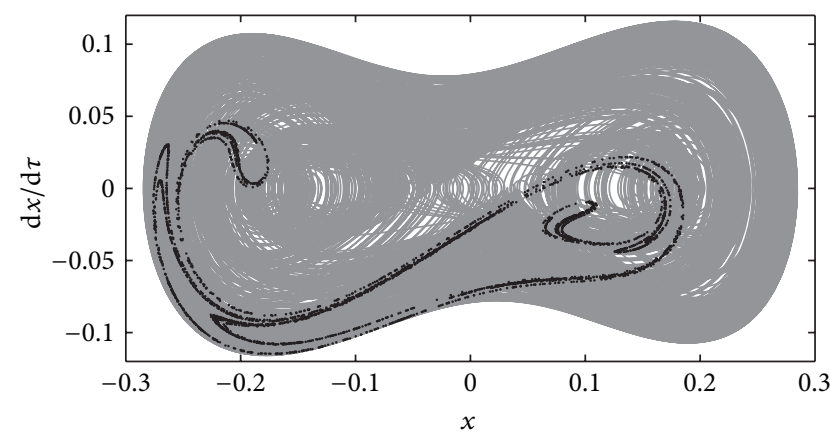

(b)

Figure 10: Chaotic response of the system for $\omega_{e}=12 \mathrm{~Hz}$ and $Z_{e}=2.24 \mathrm{~g}$ : (a) time series and frequency spectrum and (b) phase plot and Poincaré section.

with physical parameters listed in Table 1 is presented in Figure 14(b). The experimental setup fixed on a vibration exciter consists of a base, a piezoelectric beam, two magnets, and a slide. The experimental apparatus mainly includes a vibration exciter (B\&K Vibration Exciter Type 4808), a laser scanning vibrometer (Polytec PSV-400-3D), power amplifier (B\&K2720), and a data acquisition system (TST5912). The experimental process is shown in Figure 15. The excitation signal is produced by the internal signal generator of the Polytec scanning vibrometer and is transmitted through the power amplifier to the vibration exciter, so as to provide base excitation for the piezoelectric oscillator. As we know, the key factor related to power generation is relative motion response rather than absolute one. In order to acquire the relative displacement at the tip of the piezoelectric beam, we set the Polytec PSV-400-3D laser scanning vibrometer as three independent $1 \mathrm{D}$ modes (the junction box being PSV-E-40x3D (1D)) and use two scanning heads of the laser scanning vibrometer to record the vibration responses for the tip mass (magnet $A$ ) and the base of the beam. The beam is connected in series with a resistive load, and the voltage across the resistance is measured by the data acquisition system. The magnet spacing can be adjusted by rotating the screw knob located on the right side of the setup, and the screw pitch is
$1 \mathrm{~mm}$. In the following experiments, the spacing of the two magnets was held constant at $13.5 \mathrm{~mm}$.

4.2. Experimental Results. In the experiment, the amplitude of the base excitation can be set by adjusting the output voltage of the internal signal generator. We fix the excitation amplitude as $2.5 \mathrm{~g}$ and measure the responses for both the bistable system and linear system $(d=\infty)$ when gradually increasing the excitation frequency. The effective values of the output voltages with varying frequency are given in Table 3. Comparison implies that the generation performance of the bistable system is obviously superior to the linear system in the low-frequency region. From the detailed analysis, the interwell oscillation corresponds to the optimal power output, the chaotic motion is less effective, and the intrawell oscillation is the worst. The results are in good agreement with the numerical ones. Figures 16 and 17, respectively, illustrate the phase plot, frequency spectrum, and output voltage time series of the typical responses of the bistable system for different excitation frequencies. For $\omega_{e}=15 \mathrm{~Hz}$ the response is periodic with large amplitude, while for $\omega_{e}=$ $25 \mathrm{~Hz}$ the response is chaotic.

To verify the results of numerical simulations in the previous section, the experiments were carried out for increasing excitation amplitude at $\omega_{e}=12 \mathrm{~Hz}$. The asymmetric period-1 


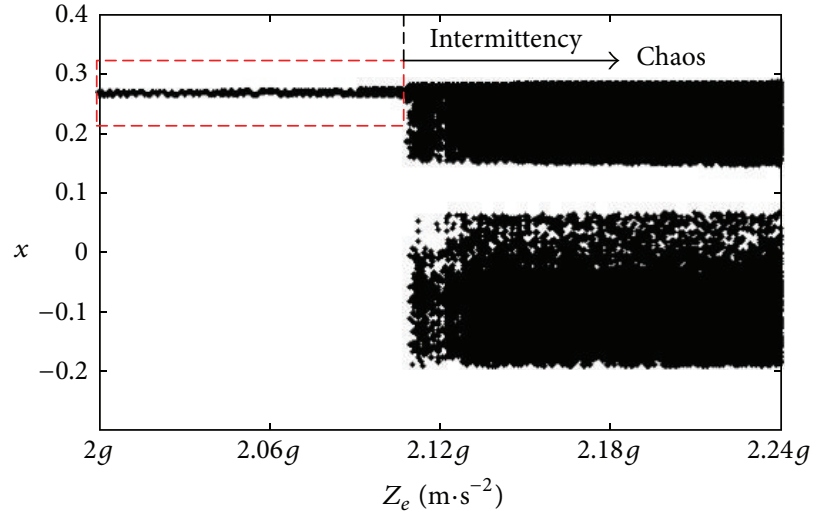

(a)

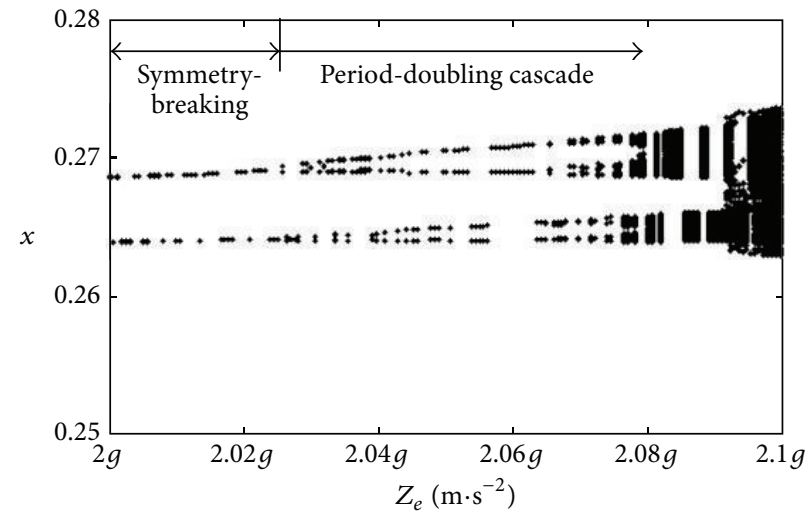

(b)

FIGURE 11: (a) Bifurcation diagram for increasing $Z_{e}$ with $\omega_{e}=12 \mathrm{~Hz}$ and (b) its locally enlarged portions for the range [2g, 2.1g].

TABLE 3: Responses of bistable generator versus linear configuration for various excitations frequencies.

\begin{tabular}{lccc}
\hline$\omega_{e}(\mathrm{~Hz})$ & $u_{\mathrm{RMS}}(\mathrm{mV})(d=13.5 \mathrm{~mm})$ & Response type $(d=13.5 \mathrm{~mm})$ & $u_{\mathrm{RMS}}(\mathrm{mV})(d=\infty)$ \\
\hline 10 & 1944 & Intrawell motion & 52 \\
15 & 5244 & Interwell period-1 & 96 \\
20 & 5347 & Interwell period-1 & 149 \\
25 & 2245 & Chaotic motion & 332 \\
35 & 1321 & Intrawell motion & 1458 \\
\hline
\end{tabular}

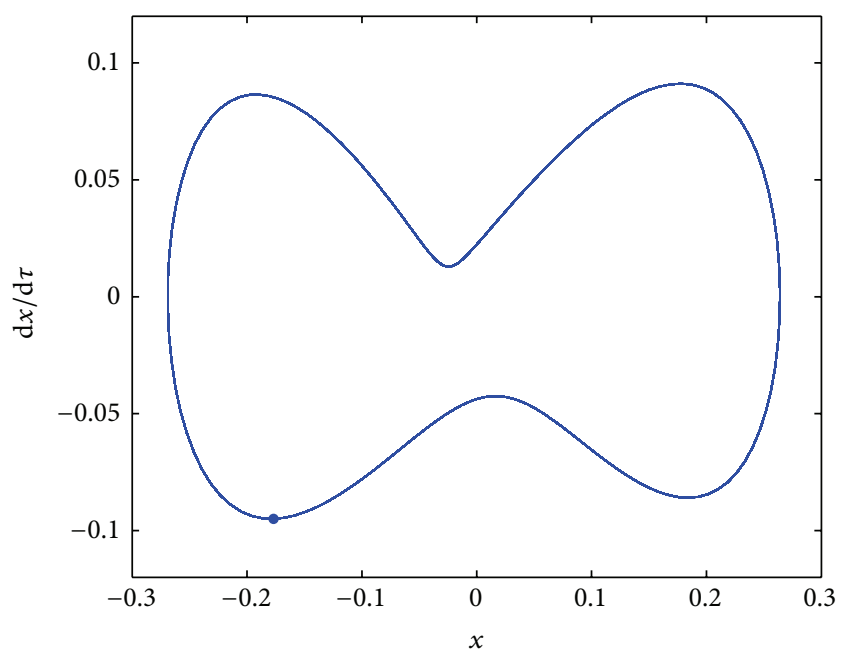

FIGURE 12: Phase plot and Poincaré section for $Z_{e}=2.02 \mathrm{~g}$.

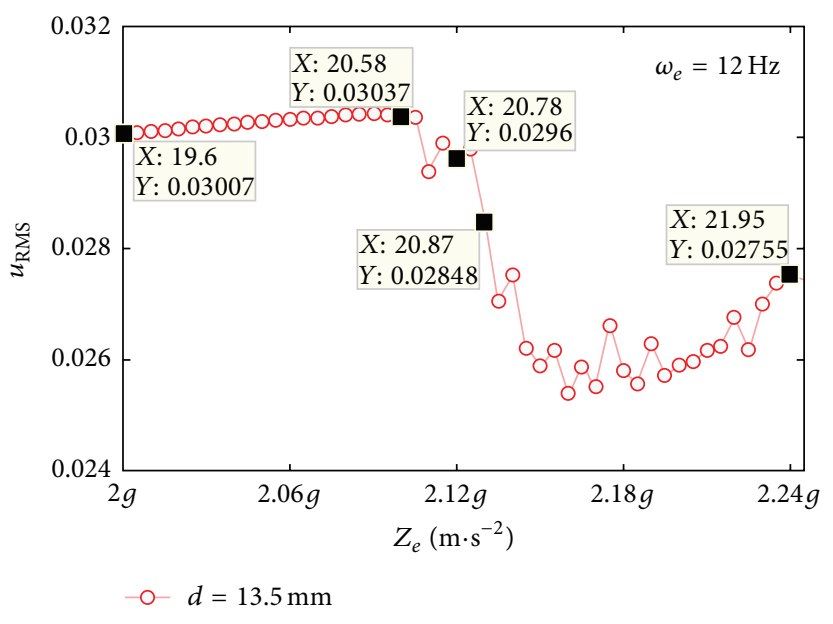

FIGURE 13: Effective voltage output with increasing $Z_{e}$. oscillation (Figure 18(a)), period-4 oscillation (Figure 18(b)), and the intermittent chaos (Figure 19(a)) are all observed as $Z_{e}$ is gradually increased. Then, a further increase in $Z_{e}$ leads to a chaotic movement, as shown in Figure 19(b). Since the full period-doubling cascade corresponds to a little range of excitation amplitude, it is difficult to observe the series of period-doubling bifurcation and transition to chaos by adjusting the output of signal generator manually. However, it is possible to observe the qualitative trend of both period-doubling and intermittency routes leading to chaos by increasing the excitation amplitude, which agrees well with the numerical analysis. Figure 20 presents the output voltage time series corresponding to the four cases of Figures 18 and 19. It is seen that the power output is not proportional to the excitation level due to the bifurcation behaviours; it does not reduce obviously when the system undergoes a sequence of period-doubling bifurcations and the beginning of intermittency, whereas the power output decreases significantly when the response turns into chaotic.

The experimental results take a good agreement with the numerical simulations qualitatively, indicating that the distributed-parameter model can reflect the real nonlinearity 


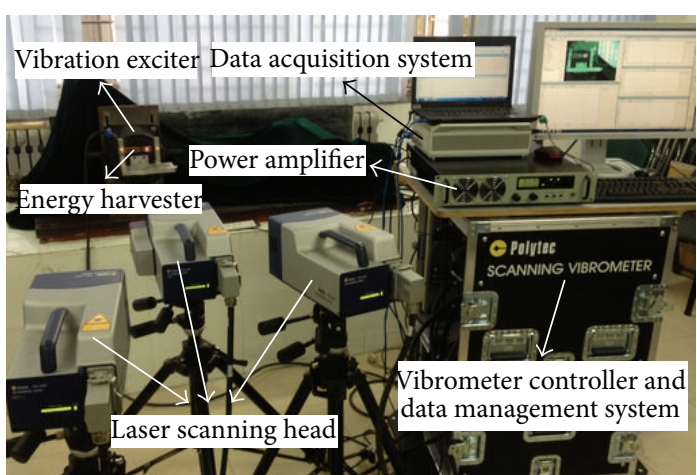

(a)

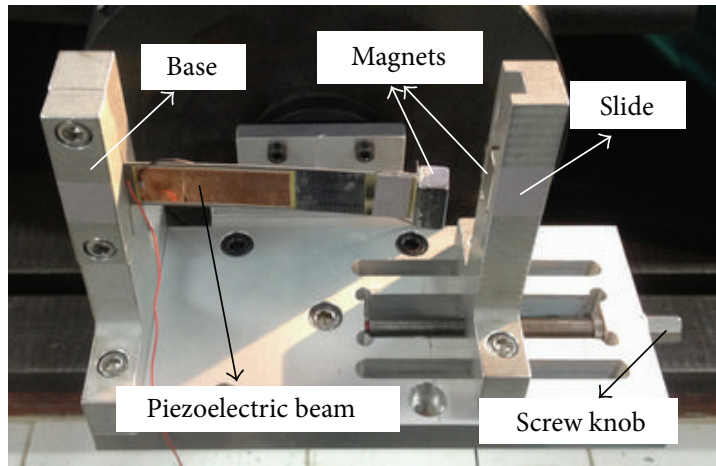

(b)

FIGURE 14: Schematic of the experimental setup: (a) overall layout and (b) bistable piezoelectric generator device.

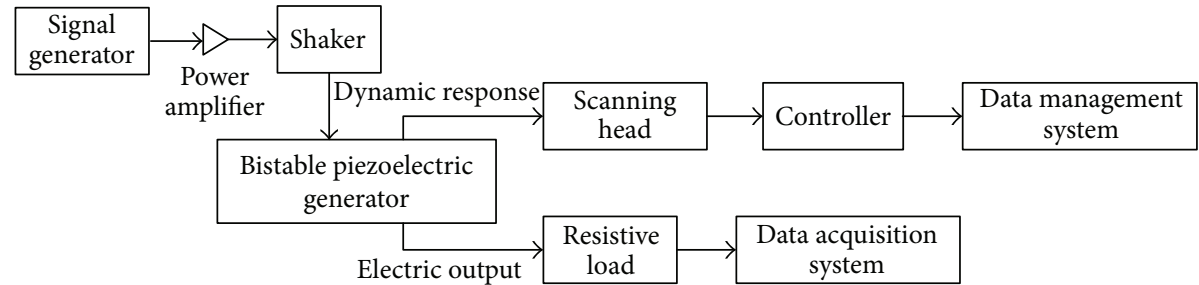

FIGURE 15: Schematic of the experimental process.

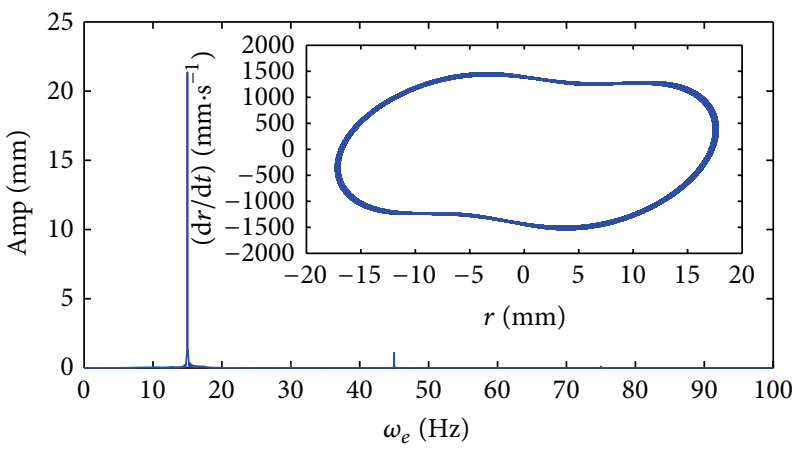

(a)

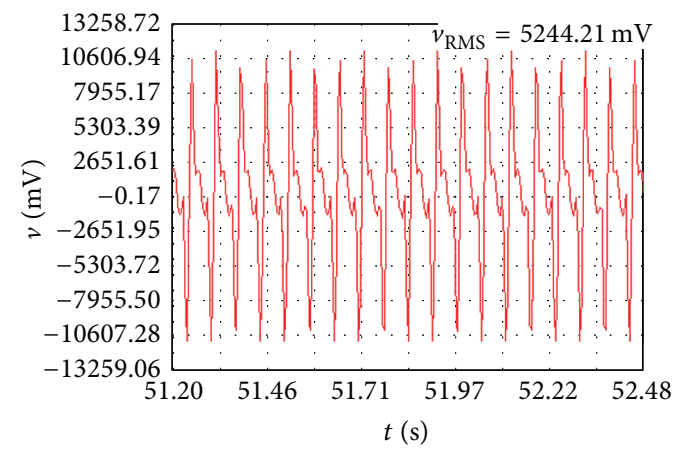

(b)

FiguRE 16: Experimentally obtained period-1 response for $Z_{e}=2.5 \mathrm{~g}$ and $\omega_{e}=15 \mathrm{~Hz}$ : (a) phase plot and frequency spectrum and (b) time series of output voltage.

and power generation law in the bistable system. In the meantime, modelling assumptions and inevitable imperfections in the experiments yield certain quantitative differences from the numerical results.

\section{Conclusions and Discussion}

Based on the proposed piezomagnetoelastic distributedparameter model of a bistable piezoelectric power generator (BPCG), both numerical and experimental studies are performed to study the dynamic responses, the bifurcation characteristics, especially the features of transition to chaos, and electricity generation performance of the BPCG. Under certain excitation frequency, with increase of excitation amplitude, several complex nonlinear dynamic behaviors are discovered in the BPCG, which include the phase-amplitudemodulation, symmetric-breaking bifurcation, coexistence of period-doubling bifurcation, and intermittency to chaos. The large amplitude limit cycle oscillation in the bistable system corresponds to the most power generation. Chaos and interwell multiperiod motion containing intrawell oscillations have lower power output. The power generation is not assured to increase with the increasing of input energy due to the presence of bifurcation. During the sequence of periodicdoubling bifurcations and the beginning of intermittency, power generation keeps at a higher level and does not change significantly. However, when the system goes into chaos, power output reduces obviously. The conclusions obtained in the paper are helpful to select the effective motions and the range of parameters for maximum power 


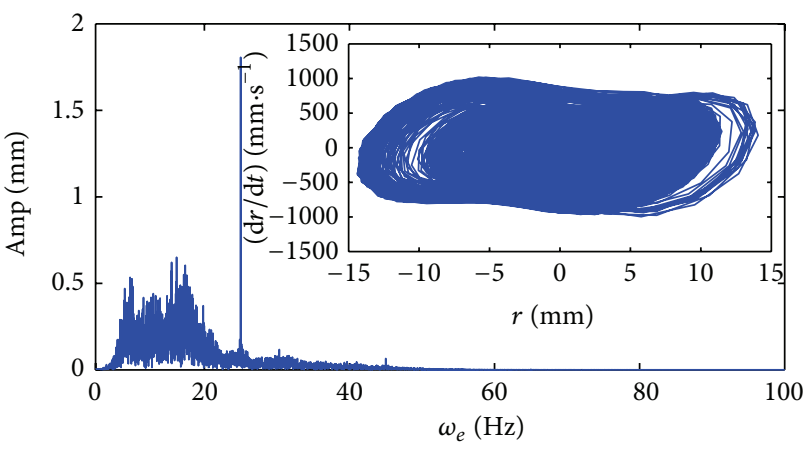

(a)

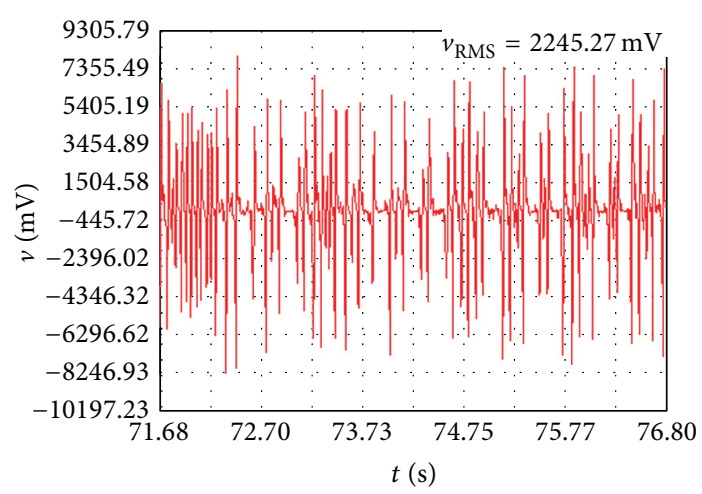

(b)

FIGURE 17: Experimentally obtained chaotic response for $Z_{e}=2.5 \mathrm{~g}$ and $\omega_{e}=25 \mathrm{~Hz}$ : (a) phase plot and frequency spectrum and (b) time series of output voltage.
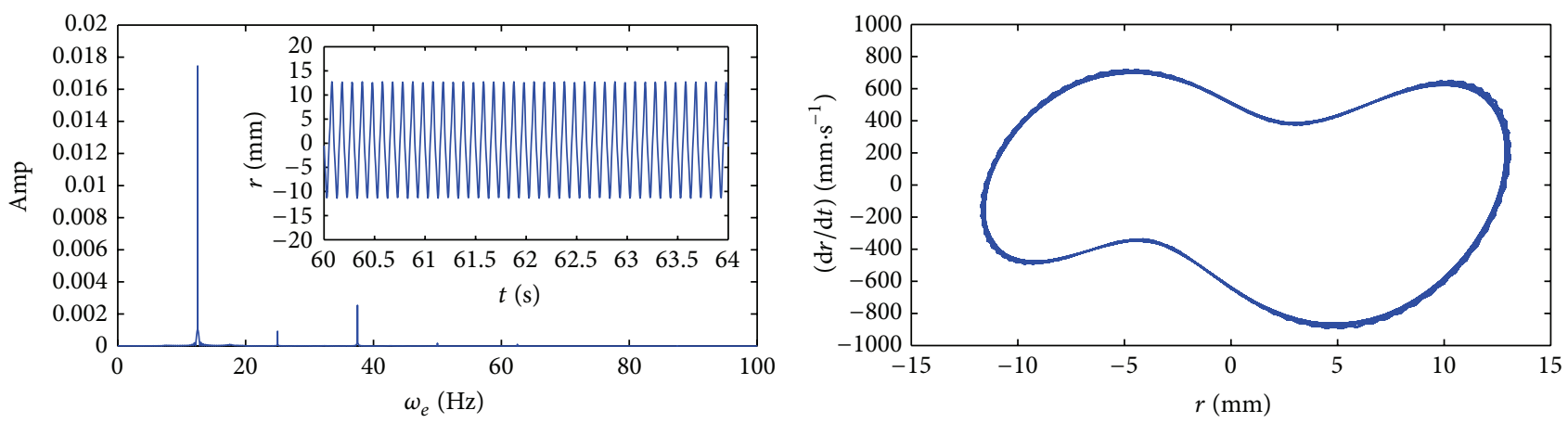

(a)
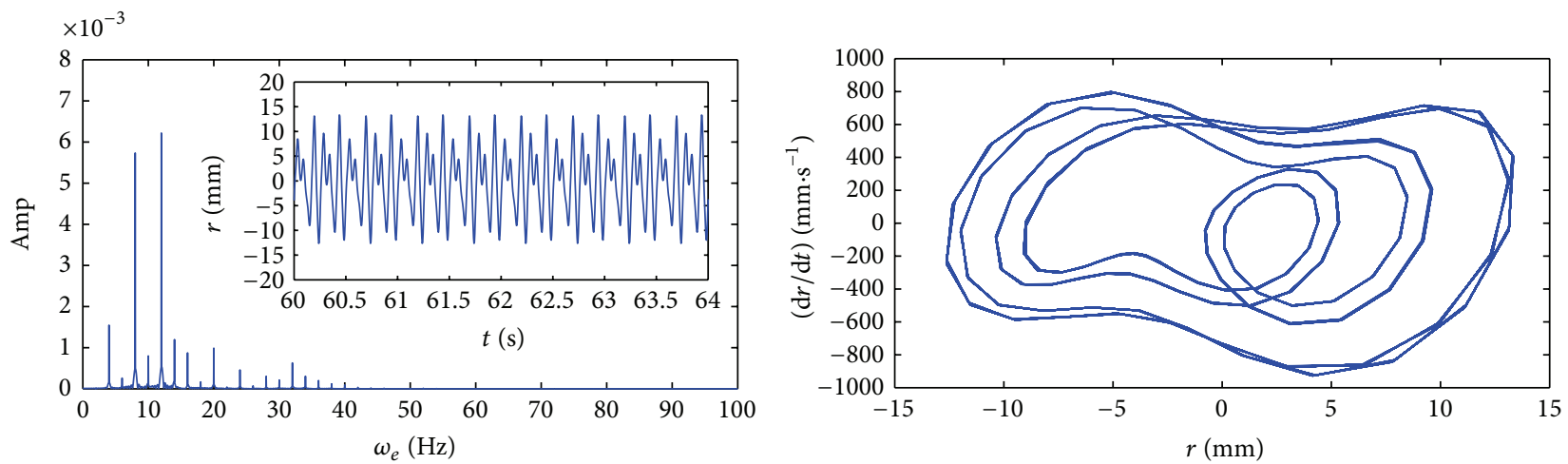

(b)

FiguRE 18: Experimentally obtained time series and frequency spectrum (left), and phase trajectories (right) for $\omega_{e}=12 \mathrm{~Hz}$ with different $Z_{e}$ : (a) $Z_{e}=2 g$ and (b) $Z_{e}=2.1 g$.

generation performance. Future work will incorporate the structural geometry nonlinear and damping nonlinear into the modeling to moderate the quantitative error between the simulation and experimental results. The effects of higher order vibration modes on the system response need further study as well. The work presented in this paper tries to explore further potentials of nonlinear energy generator at a wider low-frequency region and reveal the complicated dynamical behaviors of the proposed multiphysical field coupled system.

\section{Conflict of Interests}

The authors declare that there is no conflict of interests regarding the publication of this paper. 


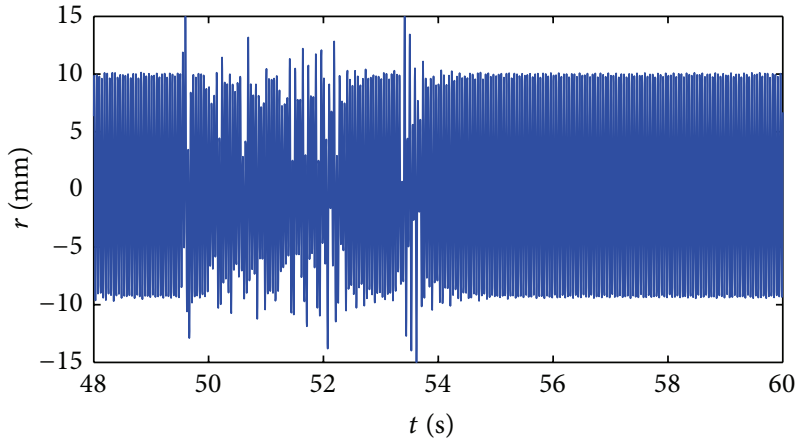

(a)

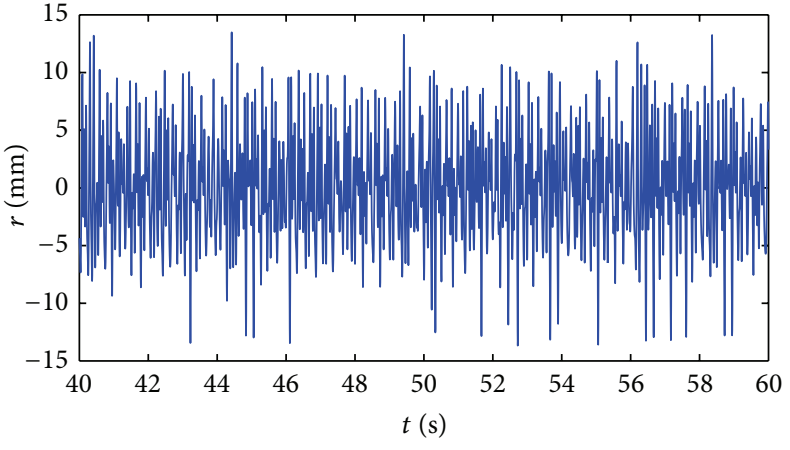

(b)

FIGURE 19: Experimentally obtained time series for $\omega_{e}=12 \mathrm{~Hz}$ with different $Z_{e}$ : (a) $Z_{e}=2.2 g$ and (b) $Z_{e}=2.3 g$.

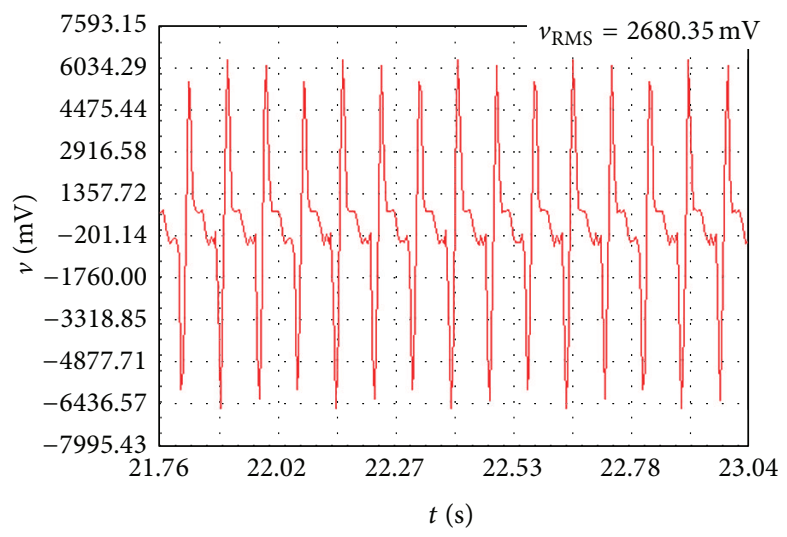

(a) $Z_{e}=2 g$

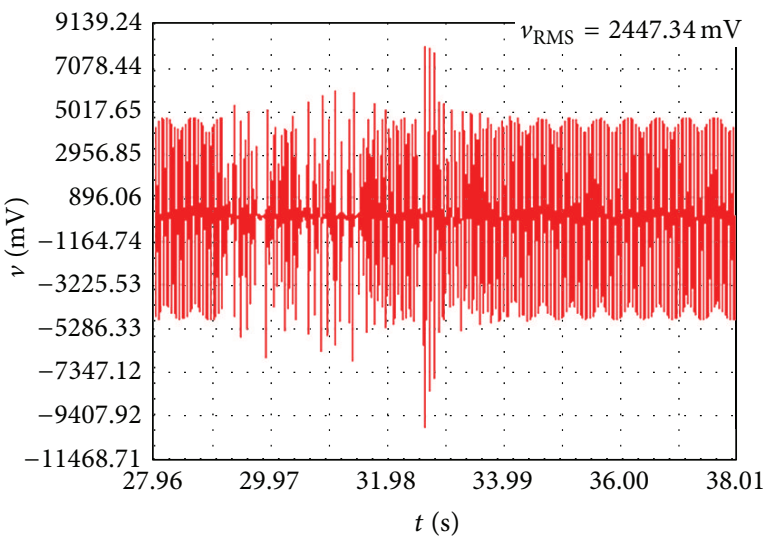

(c) $Z_{e}=2.2 g$

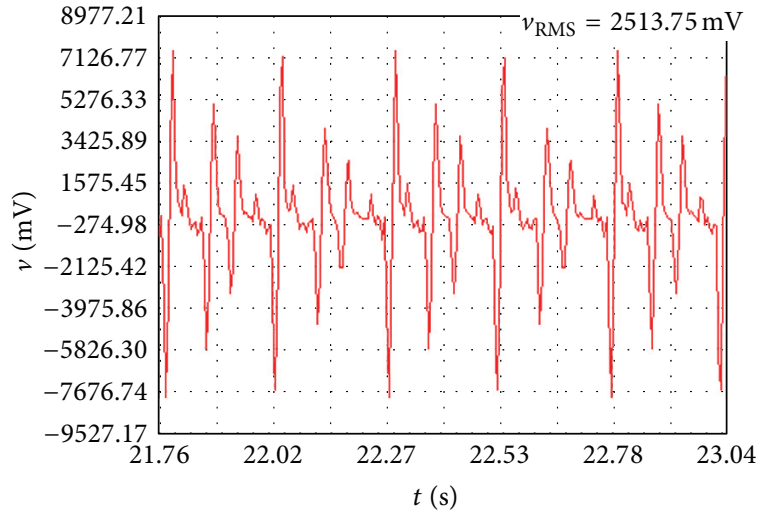

(b) $Z_{e}=2.1 \mathrm{~g}$

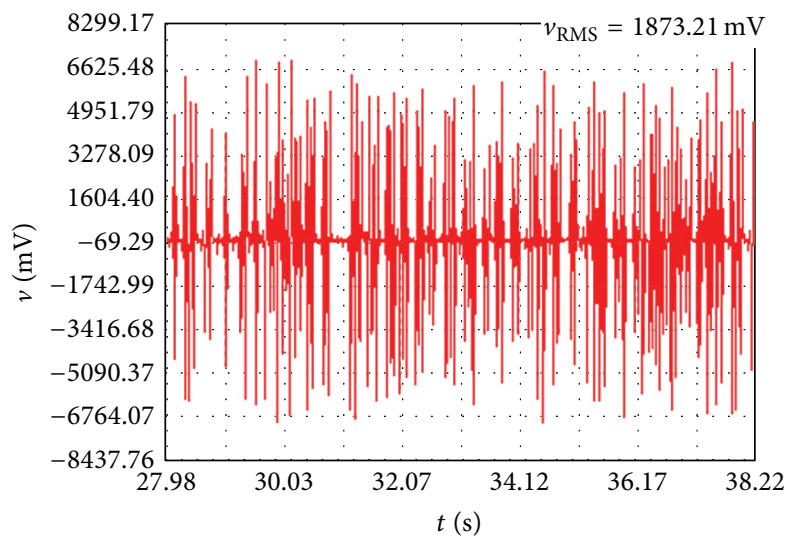

(d) $Z_{e}=2.3 g$

FiguRE 20: Experimentally obtained time series of output voltage for $\omega_{e}=12 \mathrm{~Hz}$ with different $Z_{e}$.

\section{Acknowledgments}

This work was supported by the National Natural Science Foundation of China (Grant no. 11172199) and the National Natural Science Foundation of China (Grant no. 51175370).

\section{References}

[1] S. Roundy, D. Steingart, L. Frechette, P. Wright, and J. Rabaey, "Power sources for wireless sensor networks," in Wireless Sensor
Networks, vol. 2920 of Lecture Notes in Computer Science, pp. 117, Springer, Berlin, Germany, 2004.

[2] K. A. Cook-Chennault, N. Thambi, and A. M. Sastry, "Powering MEMS portable devices-a review of non-regenerative and regenerative power supply systems with special emphasis on piezoelectric energy harvesting systems," Smart Materials and Structures, vol. 17, no. 4, Article ID 043001, 2008.

[3] S. R. Anton and H. A. Sodano, "A review of power harvesting using piezoelectric materials (2003-2006)," Smart Materials and Structures, vol. 16, no. 3, pp. R1-R21, 2007. 
[4] M. Kim, M. Hoegen, J. Dugundji, and B. L. Wardle, "Modeling and experimental verification of proof mass effects on vibration energy harvester performance," Smart Materials and Structures, vol. 19, no. 4, Article ID 045023, 2010.

[5] S. Zhao and A. Erturk, "Electroelastic modeling and experimental validations of piezoelectric energy harvesting from broadband random vibrations of cantilevered bimorphs," Smart Materials and Structures, vol. 22, no. 1, Article ID 015002, 2013.

[6] M. Rosa and C. De Marqui Junior, "Modeling and analysis of a piezoelectric energy harvester with varying cross-sectional area," Shock and Vibration, vol. 2014, Article ID 930503, 9 pages, 2014.

[7] M. Ferrari, V. Ferrari, M. Guizzetti, D. Marioli, and A. Taroni, "Piezoelectric multifrequency energy converter for power harvesting in autonomous microsystems," Sensors and Actuators A: Physical, vol. 142, no. 1, pp. 329-335, 2008.

[8] H. Xue, Y. Hu, and Q.-M. Wang, "Broadband piezoelectric energy harvesting devices using multiple bimorphs with different operating frequencies," IEEE Transactions on Ultrasonics, Ferroelectrics, and Frequency Control, vol. 55, no. 9, pp. 21042108, 2008.

[9] X. Wu, J. Lin, S. Kato, K. Zhang, T. Ren, and L. Liu, "A frequency adjustable vibration energy harvester," in Proceedings of the PowerMEMS, pp. 245-248, 2008.

[10] C. Eichhorn, F. Goldschmidtboeing, and P. Woias, "Bidirectional frequency tuning of a piezoelectric energy converter based on a cantilever beam," Journal of Micromechanics and Microengineering, vol. 19, no. 9, Article ID 094006, 2009.

[11] S. Burrow, L. Clare, A. Carrella, and D. Barton, "Vibration energy harvesters with non-linearcompliance," in Proceedings of the 15th International Symposium on Smart Structures and Materials \& Nondestructive Evaluation and Health Monitoring, San Diego, Calif, USA, April 2008.

[12] B. P. Mann and N. D. Sims, "Energy harvesting from the nonlinear oscillations of magnetic levitation," Journal of Sound and Vibration, vol. 319, no. 1-2, pp. 515-530, 2009.

[13] S. C. Stanton, C. C. McGehee, and B. P. Mann, "Reversible hysteresis for broadband magnetopiezoelastic energy harvesting," Applied Physics Letters, vol. 95, no. 17, Article ID 174103, 2009.

[14] L. Gammaitoni, P. Hänggi, P. Jung, and F. Marchesoni, "Stochastic resonance: a remarkable idea that changed our perception of noise," European Physical Journal B-Condensed Matter and Complex Systems, vol. 69, no. 1, pp. 1-3, 2009.

[15] A. Erturk, J. Hoffmann, and D. J. Inman, "A piezomagnetoelastic structure for broadband vibration energy harvesting," Applied Physics Letters, vol. 94, no. 25, Article ID 254102, 2009.

[16] F. C. Moon and P. J. Holmes, "A magnetoelastic strange attractor," Journal of Sound and Vibration, vol. 65, no. 2, pp. 275296, 1979.

[17] A. F. Arrieta, P. Hagedorn, A. Erturk, and D. J. Inman, "A piezoelectric bistable plate for nonlinear broadband energy harvesting," Applied Physics Letters, vol. 97, no. 10, Article ID 104102, 2010.

[18] M. Ferrari, V. Ferrari, M. Guizzetti, B. Andò, S. Baglio, and C. Trigona, "Improved energy harvesting from wideband vibrations by nonlinear piezoelectric converters," Sensors and Actuators A: Physical, vol. 162, no. 2, pp. 425-431, 2010.

[19] R. Ramlan, M. J. Brennan, B. R. MacE, and I. Kovacic, "Potential benefits of a non-linear stiffness in an energy harvesting device," Nonlinear Dynamics, vol. 59, no. 4, pp. 545-558, 2010.
[20] S. C. Stanton, C. C. McGehee, and B. P. Mann, "Nonlinear dynamics for broadband energy harvesting: investigation of a bistable piezoelectric inertial generator," Physica D: Nonlinear Phenomena, vol. 239, no. 10, pp. 640-653, 2010.

[21] S. C. Stanton, B. P. Mann, and B. A. M. Owens, "Melnikov theoretic methods for characterizing the dynamics of the bistable piezoelectric inertial generator in complex spectral environments," Physica D: Nonlinear Phenomena, vol. 241, no. 6, pp. 711-720, 2012.

[22] S. C. Stanton, B. A. M. Owens, and B. P. Mann, "Harmonic balance analysis of the bistable piezoelectric inertial generator," Journal of Sound and Vibration, vol. 331, no. 15, pp. 3617-3627, 2012.

[23] J.-T. Lin and B. Alphenaar, "Enhancement of energy harvested from a random vibration source by magnetic coupling of a piezoelectric cantilever," Journal of Intelligent Material Systems and Structures, vol. 21, no. 13, pp. 1337-1341, 2010.

[24] S. Sun and S.-Q. Cao, "Dynamic modeling and analysis of a bistable piezoelectric cantilever power generation system," Acta Physica Sinica, vol. 61, no. 21, Article ID 210505, 2012.

[25] L. Tang, Y. Yang, and C.-K. Soh, "Improving functionality of vibration energy harvesters using magnets," Journal of Intelligent Material Systems and Structures, vol. 23, no. 13, pp. 1433-1449, 2012.

[26] C. R. McInnes, D. G. Gorman, and M. P. Cartmell, "Enhanced vibrational energy harvesting using nonlinear stochastic resonance," Journal of Sound and Vibration, vol. 318, no. 4-5, pp. 655662, 2008.

[27] S. P. Pellegrini, N. Tolou, M. Schenk, and J. L. Herder, "Bistable vibration energy harvesters: a review," Journal of Intelligent Material Systems and Structures, vol. 24, no. 11, pp. 1303-1312, 2013.

[28] R. L. Harne and K. W. Wang, "A review of the recent research on vibration energy harvesting via bistable systems," Smart Materials and Structures, vol. 22, no. 2, Article ID 023001, 2013.

[29] K. W. Yung, P. B. Landecker, and D. D. Villani, "An analytic solution for the force between twomagnetic dipoles," Magnetic and Electrical Separation, vol. 9, pp. 39-52, 1998.

[30] I. Kovacic and M. J. Brennan, The Duffing Equation: Nonlinear Oscillators and Their Behaviour, John Wiley \& Sons, Chichester, UK, 2011. 

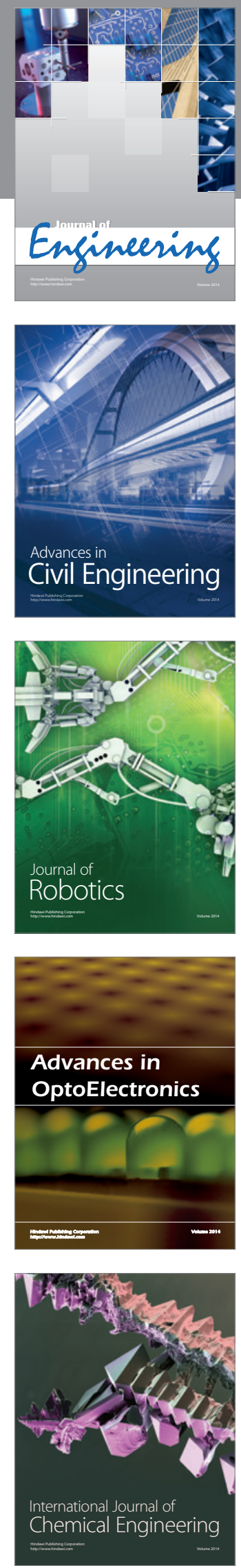

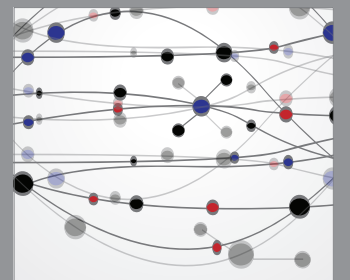

The Scientific World Journal
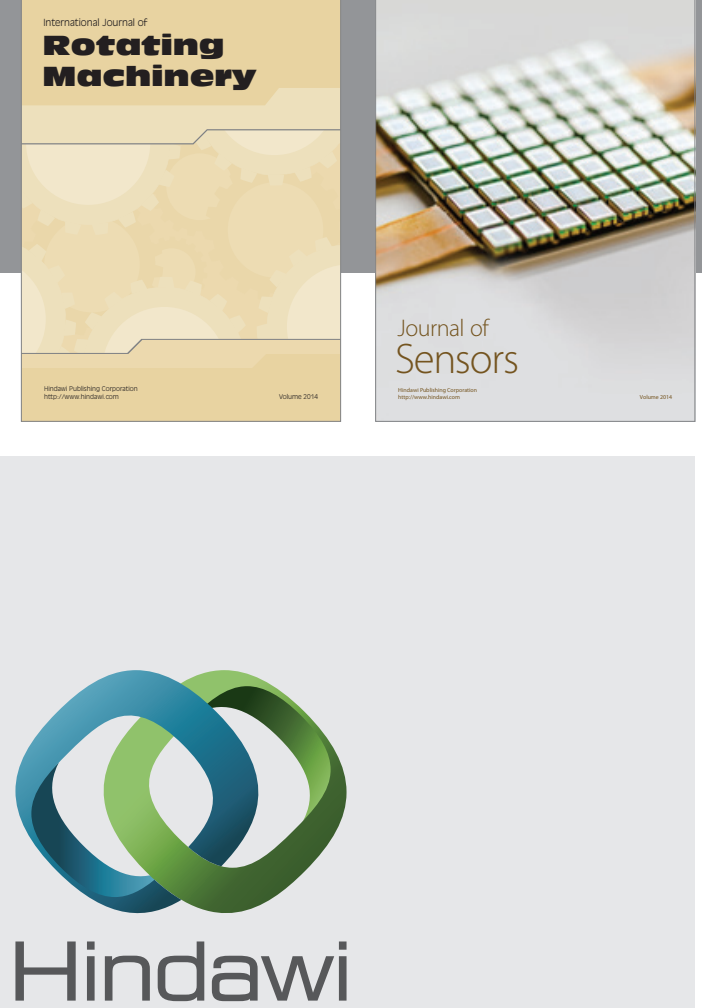

Submit your manuscripts at http://www.hindawi.com
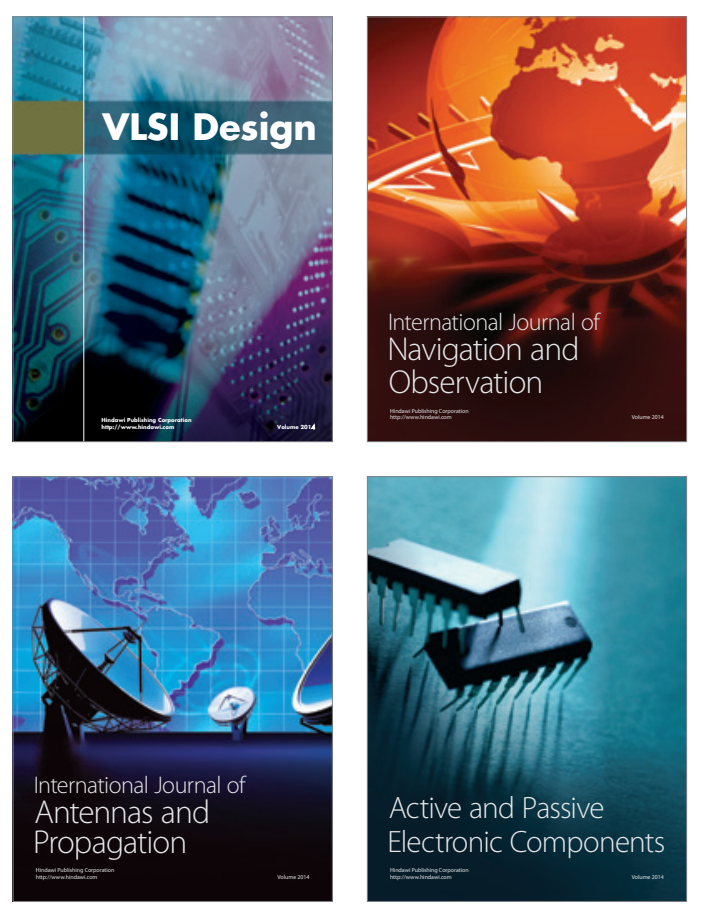
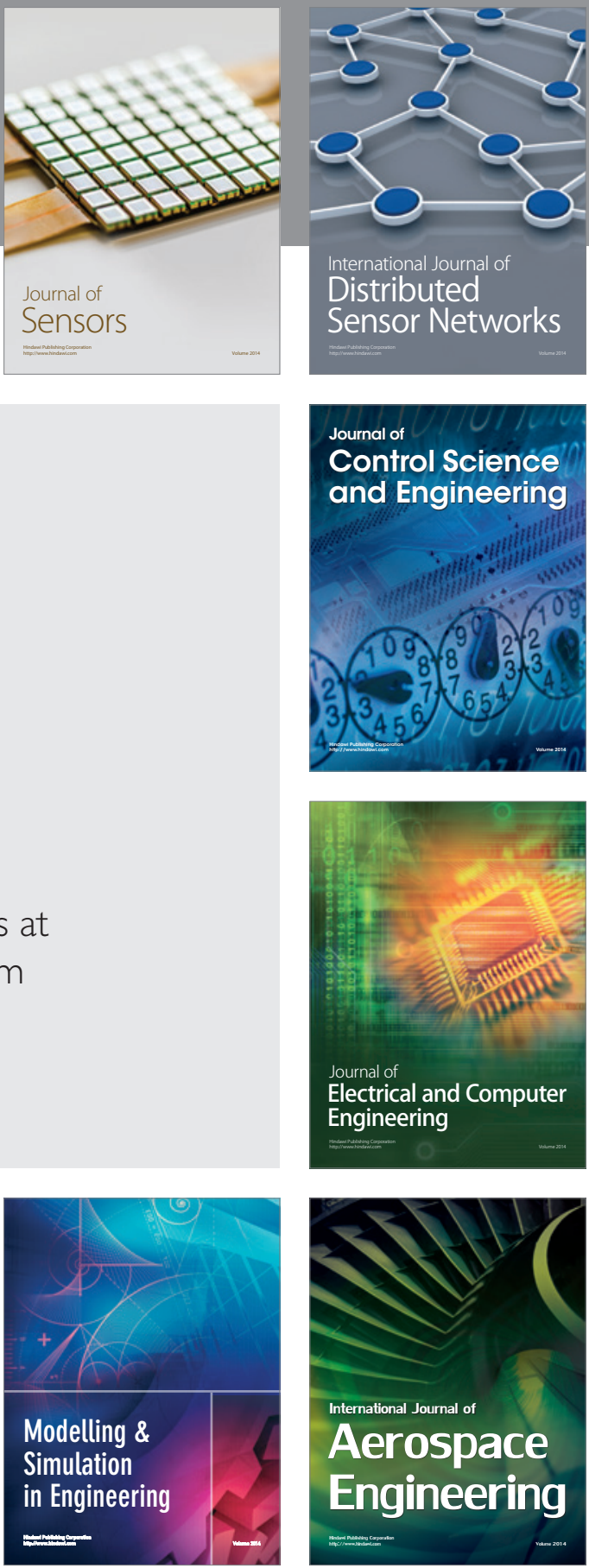

Journal of

Control Science

and Engineering
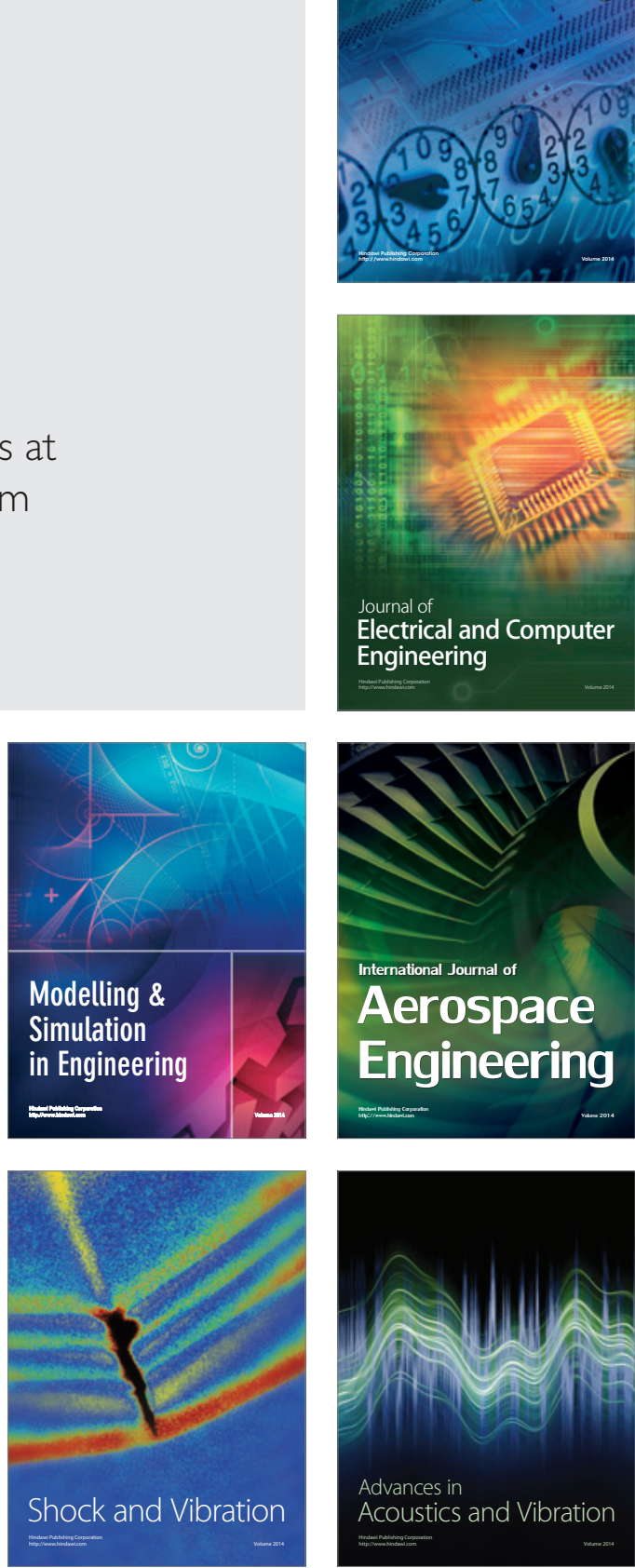